\title{
Raman scattering in symbiotic stars. II. Numerical models
}

\author{
T.J. Harries ${ }^{1}$ and I.D. Howarth ${ }^{2}$ \\ 1 School of Physics and Astronomy, University of St. Andrews, North Haugh, St. Andrews, Fife KY16 9SS, Scotland \\ 2 Dept. of Physics and Astronomy, University College London, Gower Street, London WC1E 6BT, UK
}

Received December 27, 1995; accepted April 20, 1996

\begin{abstract}
We present numerical models of Raman-line formation in symbiotic systems, based on Monte-Carlo simulations of scattering of O VI resonance-line photons from a hot source in the extended, expanding atmosphere of a cool companion, and we explore the sensitivity of the model results to the input parameters. The model successfully reproduces the observed line ratios, velocities, and polarizations, and the viewing-angle (orbital) dependence of the line strengths and polarized intensities. In principle, the position-angle dependence of the polarization in resolved Raman lines provides a diagnostic of the extent of the ionized region in symbiotic systems; however, axisymmetric geometries with 'up-down' symmetry, such as we use, fail to reproduce the most commonly observed dependence of PA on velocity. Raman-line polarization morphology is shown to be a powerful probe of the conditions in the red-giant wind; this is particularly true if observations are available at different orbital phases, so that geometrical and physical variables may more easily be isolated.
\end{abstract}

Key words: stars: binaries: symbiotic — stars: mass-loss — polarization

\section{Introduction}

Symbiotic stars are binary systems comprising a hot component which ionizes material shed by a cool, lowgravity companion. The resulting 'composite' spectra contain sharp nebular lines superimposed on a red-star spectrum, which usually includes molecular absorption bands. Two of the optical emission lines $-\lambda 6825 \AA$ and $\lambda 7082 \AA$ - have been identified as resulting from inelastic Raman scattering of $\mathrm{O}$ VI $\lambda 1032,1038 \AA$ photons off neutral hydrogen in the cool stellar wind (Schmid 1989). These lines are highly polarized (Schmid \& Schild 1990,

Send offprint requests to: T.J. Harries (tjh@st-and.ac.uk)
1994) because of the asymmetric nature of the scattering geometry, and intermediate-dispersion spectropolarimetry shows that the $\lambda \lambda 6825,7082 \AA$ emission lines display a range of complex intensity and polarization characteristics which demonstrate that the scattering must take place in an extended, expanding cool-star wind (e.g., Harries \& Howarth 1996b, hereafter Paper I).

While many mass-loss tracers, such as $\mathrm{CO}$ or dust, represent minority species by mass, the $\lambda \lambda 6825,7082 \AA$ lines result from scattering off neutral hydrogen - a major component of the wind. The polarization profiles of the Raman lines ${ }^{1}$ encode both velocity and spatial information; it is the aim of this work to develop models which aid in the interpretation of spectropolarimetric observations, with the long-term goals of constraining both mass-loss rates and velocity laws for red-giant winds, for a variety of subtypes, and of obtaining binary orbital parameters for the systems.

The first attempt to model the polarization properties of symbiotic systems was carried out by Schmid (1992), who adopted a geometry of rotational symmetry about the binary axis, with a photon source illuminating a geometrically thin red-giant photosphere. The free parameters of the model were the binary separation and the absorption cross-sections for O vi photons and Raman-scattered photons. Schmid found that his model is able to reproduce observed $I_{\lambda 6825} / I_{\lambda 7082}$ line-intensity ratios, and that the predicted line polarizations obtained are comparable with observations.

The major simplification of Schmid's work was the assumption of a 'billiard ball' geometry for the red-giant component. Subsequent observations (e.g., Paper I) have demonstrated that the polarization profiles are highly

1 Because the final state of the scattering system has greater energy than the initial state, the observed spectral features are, strictly, Stokes lines (e.g., Bransden \& Joachain 1983). We will refer to them as Raman lines, both because this has become the established terminology in astronomical applications, and to avoid possible confusion which may result from the separate requirement of discussing the Stokes parameters. 
structured; the presence of this structure shows that it is essential to take account of the extension of the redgiant atmosphere, and of the velocity gradients within it, to model the data properly. The code described in the following sections treats the polarized radiative transfer by following multiple Rayleigh and Raman scatterings in a spherically symmetric wind and stellar photosphere.

\section{Model formulation}

We have followed Schmid in using Monte-Carlo techniques to model the line formation. This is in part because the Raman line-formation process is a multi-scattering problem, and does not lend itself easily to an analytical treatment; but also because the flexibility which the MonteCarlo approach offers in formulating different versions of the model. The problem is therefore simply one of generating source $\mathrm{O}$ VI photon packets and following the subsequent interaction of this radiation with the red-giant wind.

\subsection{Model geometry}

The model consists of a giant star, radius $R_{\mathrm{g}}$, which is centred at the origin of the Cartesian frame, and a source of the parent photons which is centred on the $x$-axis at a distance $R_{\mathrm{g}} q_{\mathrm{sep}}$ from the origin. The observer is located in the $x y$-plane, with the $z$-axis perpendicular to the lineof-sight. (This entails no loss of generality; the $x y$ plane need not be the orbital plane.)

The giant star has an extended atmosphere which is assumed to be spherically symmetric. For a mass-losing star the density structure is defined by the stellar mass-loss rate $\dot{M}$ and a velocity structure $v(r)$ through the equation of mass conservation:

$\rho(r)=\dot{M} / 4 \pi r^{2} v(r)$.

Other structures (e.g., extended, hydrostatic atmospheres) are trivially substituted.

\subsection{Stokes parameters}

A photon packet is fully described by its frequency $\nu$, position $\left(x_{0}, y_{0}, z_{0}\right)$, direction $\left(u_{0}, v_{0}, w_{0}\right)$, and normalized linear Stokes vector $(w, \bar{q}, \bar{u})$, where $w$ is the weight (initially $w \equiv 1$ ). The total Stokes parameters for $N$ photon packets are

$I=\sum_{i=1}^{N} w_{i} \quad Q=\sum_{i=1}^{N} q_{i} w_{i} \quad U=\sum_{i=1}^{N} u_{i} w_{i}$

The Stokes parameters are calculated with respect to the meridional plane containing the photon path and the $z$-axis, so the upper-lower symmetry of the system about the line-of-centres means that the $U$ Stokes parameter cancels in the integrated spectrum.

\subsection{Photon initialization}

For most models we assumed a point source of O VI photons (trial calculations for an extended source are reported in Sect. 11). We assume that the emission is isotropic, so that the initial direction of the photon packet may be obtained from

$$
\begin{aligned}
& w_{0}=2 U_{\mathrm{R}}(1)-1 \\
& u_{0}=t_{0} \cos \phi \\
& v_{0}=t_{0} \sin \phi
\end{aligned}
$$

where

$t_{0}=\sqrt{1-w_{0}^{2}}$

$\phi=2 \pi U_{\mathrm{R}}(2)$,

and $U_{\mathrm{R}}$ is a uniform random deviate in the range $0-1$.

In principle, a weighting scheme could be used to bias the initial direction of the photon packet towards the redgiant component (while retaining the assumption of intrinsically isotropic emission). We experimented with such schemes, which can improve the statistical efficiency of the calculation, but found that the high weights given to the relatively few packets emitted in the direction away from the red giant, modified by the weighting schemes discussed below, gave cosmetically unsatisfactory results for relatively small savings in computer time.

The radiation from the source region is assumed to be initially unpolarized, so that the position angle $\gamma$ of a source photon packet is given by

$\gamma=\pi U_{\mathrm{R}}$

\subsection{The random walk}

The probability of a collision event (scattering or absorption) is defined by the optical depth $\tau$, where the probability density function for an event is

$\rho(\tau)=\exp (-\tau)$.

The normalized cumulative probability function is

$$
\begin{aligned}
P\left(\tau_{l}\right) & =\int_{0}^{\tau_{l}} \exp (-\tau) \mathrm{d} \tau / \int_{0}^{\infty} \exp (-\tau) \mathrm{d} \tau \\
& =1-\exp \left(-\tau_{l}\right)
\end{aligned}
$$

where $\tau_{l}$ is the optical depth to the collision. Using the inverse transform method gives

$\tau_{l}=-\ln \left(1-U_{R}\right)$.

Once the optical depth from the photon source to the event has been obtained, the physical path length to the event is calculated. The optical depth is the integral

$\tau_{l}=\int_{0}^{l} \rho(s) \kappa \mathrm{d} s$ 
where $l$ is the path length, $\rho(s)$ the density, and $\kappa$ the total opacity. By inverting this integral equation it is possible to obtain the path length for a given optical depth; the inversion can be carried out analytically for a number of simple density distributions.

The absorption cross-section for the O vi photons, $\kappa_{\mathrm{UV}}$, and the absorption cross-section for the Ramanscattered photons, $\kappa_{\text {red }}$, may be calculated in principle, but here are treated as free parameters, specified in units of the total scattering cross-section $\sigma_{\text {tot }}$, where

$\sigma_{\text {tot }}=\sigma_{\text {Ray }}+\sigma_{\text {Ram }}$

and where $\sigma_{\text {Ray }}$ and $\sigma_{\text {Ram }}$ are the Rayleigh- and Ramanscattering cross-sections, respectively. All these quantities (especially the absorption cross-sections) are wavelengthdependent, but we adopt representative monochromatic values at the line wavelengths; in particular, we use Raman and Rayleigh scattering cross-sections from Schmid (1989).

Since better statistics are obtained as more Raman photons escape to the observer, it is inefficient to lose photons through absorption. We therefore employ a variancereduction technique in which all photon packets are forced to scatter. To conserve intensity, the photon packets are re-weighted at each scattering by a factor

$f_{1}=\exp \left(-\tau\left[\kappa_{\text {abs }} / \kappa_{\text {tot }}\right]\right)$

where $\tau$ is the scattering optical depth, $\kappa_{\text {abs }}$ is the absorption optical depth and $\kappa_{\text {tot }}$ is the total (absorption plus scattering) collision cross-section.

For additional efficiency, all O VI photons are forced to scatter before reaching the system boundary (which is taken as $1000 R_{\mathrm{g}} q_{\mathrm{sep}}$ in the numerical models presented here); i.e., all O VI photons are converted to Raman photons. If $\tau_{\infty}$ is the optical depth to the system boundary then the optical depth to scattering, $\tau_{l}$, is

$\tau_{l}=-\ln \left[1-U_{\mathrm{R}}\left(1-\exp \left(-\tau_{\infty}\right)\right)\right]$

and the photon weight, $w$, is adjusted by a factor

$f_{2}=1-\exp \left(-\tau_{\infty}\right)$

\subsection{The scattering event}

A comprehensive account of radiation transfer according to the Rayleigh-scattering phase matrix is given by Chandrasekhar (1960). The geometry for the scattering event is given in Fig. 1.

The photon packet, initially travelling in direction $O P_{1}$, is scattered through an angle $\Theta$ and exits the scattering event travelling in direction $O P_{2}$. The Stokes parameters of the incoming photon beam refer to the meridian plane $O P_{1} Z$. In order to calculate the resultant polarization the Stokes parameters must be rotated though an angle $-i_{1}$ in order that they refer to the scattering plane
$O P_{1} P_{2}$. The rotation matrix $\mathbf{L}$ that rotates the Stokes parameters through a clockwise angle $\phi$ is

$\mathbf{L}(\phi)=\left(\begin{array}{ccc}1 & 0 & 0 \\ 0 & \cos 2 \phi & \sin 2 \phi \\ 0 & -\sin 2 \phi & \cos 2 \phi\end{array}\right)$.

Thus the Stokes vector relative to the scattering plane, $\mathbf{I}_{\mathbf{s}}$, is given by

$\mathbf{I}_{\mathbf{s}}=\mathbf{L}\left(-i_{1}\right) \mathbf{I}$,

where

$\mathbf{I}=\left(\begin{array}{c}I \\ Q \\ U\end{array}\right)$

Both Raman and Rayleigh scatterings occur according to the Rayleigh-scattering phase matrix $\mathbf{R}$, where

$\mathbf{R}=\frac{3}{4}\left(\begin{array}{ccc}\cos ^{2} \theta+1 & \cos ^{2} \theta-1 & 0 \\ \cos ^{2} \theta-1 & \cos ^{2} \theta+1 & 0 \\ 0 & 0 & 2 \cos \theta\end{array}\right)$

and $\theta$ is the scattering angle. Once the phase matrix has been applied, the Stokes vector must be rotated by $\left(\pi-i_{2}\right)$ so that it refers to the meridian plane $\mathrm{OP}_{2} Z$. Thus the Stokes vectors of the incident and scattered beam, $\mathbf{I}$ and $\mathbf{I}^{\prime}$, are related by

$\mathbf{I}^{\prime}=\frac{\sigma}{4 \pi} \mathbf{L}\left(\pi-i_{2}\right) \mathbf{R} \mathbf{L}\left(-i_{1}\right) \mathbf{I}$

where $\sigma$ is the scattering cross-section.

If $\phi_{\mathrm{s}}$ is the azimuthal scattering angle measured relative to the plane of scattering then the cumulative probability distribution of the scattering angles $P\left(\theta_{\mathrm{s}}, \phi_{\mathrm{s}}\right)$ is obtained by integrating the Stokes $I$ component over all solid angles. Hence

$P\left(\theta_{\mathrm{s}}, \phi_{\mathrm{s}}\right)=\int_{0}^{\theta_{\mathrm{s}}} \int_{0}^{\phi_{\mathrm{s}}} I(\theta, \phi) \mathrm{d} \phi \mathrm{d} \theta$.

This two-dimensional equation must be decomposed into two one-dimensional equations (cf. Schmid 1992). Integrating Eq. (21) over $\phi$ gives the cumulative probability distribution function

$P\left(\theta_{\mathrm{s}}\right)=\int_{0}^{\theta_{\mathrm{s}}} \frac{3}{8}\left(\cos ^{2} \theta+1\right) \sin \theta \mathrm{d} \theta$,

while integrating over $\theta$, and using the normalization

$\int_{-\pi}^{\pi} \rho_{\theta}(\phi) \mathrm{d} \phi=1$

gives the cumulative probability distribution function

$P_{\theta}(\phi)=\frac{1}{2 \pi} \int_{-\pi}^{\phi_{\mathrm{s}}} 1+\frac{\cos ^{2} \theta_{\mathrm{s}}-1}{\cos ^{2} \theta_{\mathrm{s}}+1} \cos 2 \phi \mathrm{d} \phi$. 


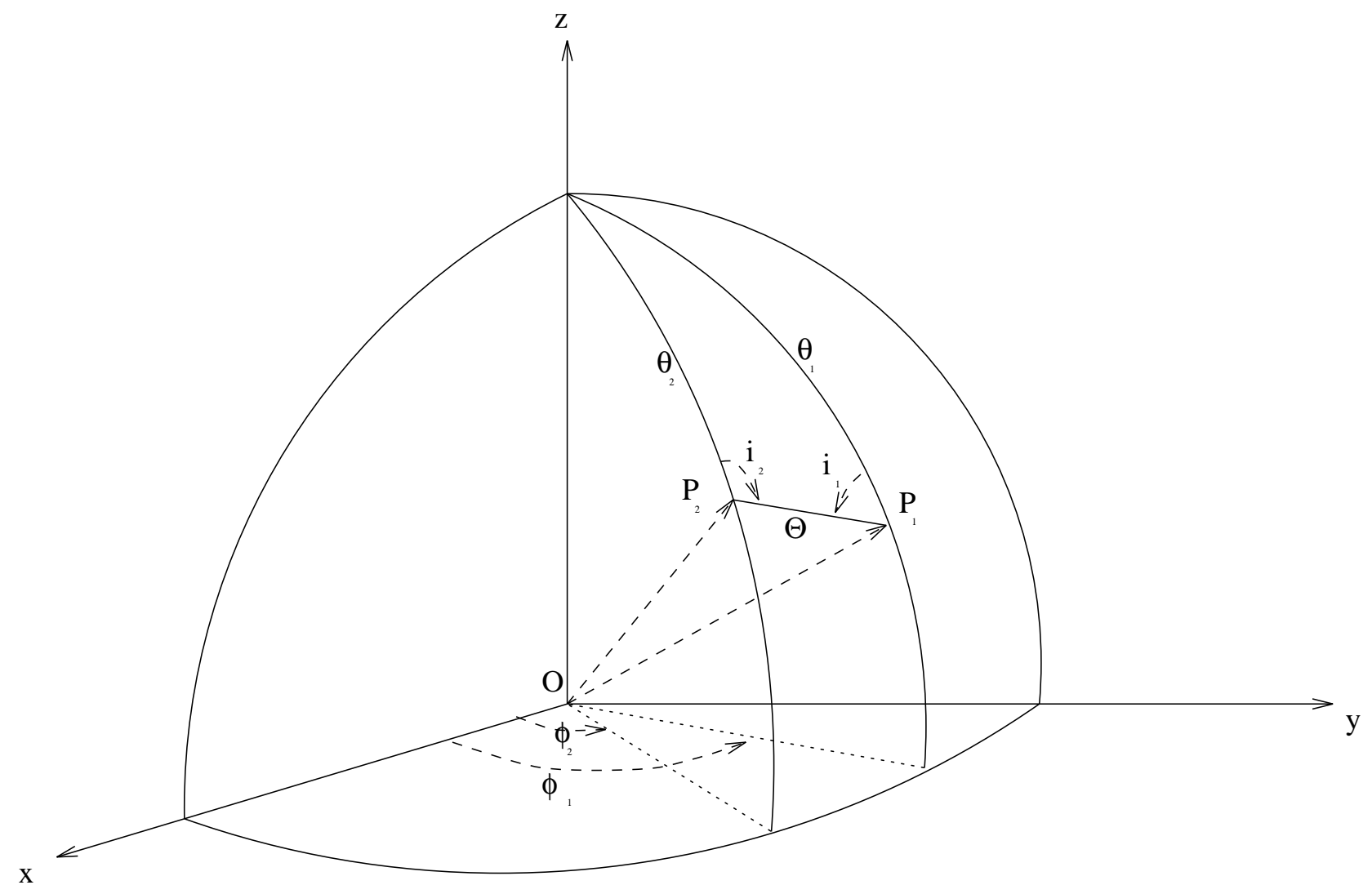

Fig. 1. The geometry of a scattering event. The photon packet encounters the scatterer at $O$, entering while travelling in the direction $O P_{1}$ and exiting in the direction $O P_{2}$, having been scattered through an angle $\theta$

The inverse-transform method was used to set up look-up tables of $\left(\theta_{\mathrm{s}}, \phi_{\mathrm{s}}\right)$. For Rayleigh-scattering events, the new photon direction with respect to the scattering plane is found from interpolation in these tables. The new photon-packet direction in the Cartesian frame is then obtained from trigonometric relations. The polarization with respect to the new photon-packet direction is obtained by applying Eq. (20) to the incident polarization vector. The intensity component of the Stokes vector is not altered, as the intensity distribution of the phase matrix is included in the choice of photon-packet direction.

When a Raman-scattering event occurs, the photon packet is forced to scatter towards the observer. The new polarization vector is again found by applying Eq. (20). The photon packet must be weighted by the probability that it is scattered towards to observer, so that the final weight of a packet at the $n$th scattering is

$w_{n}=w_{n-1} f_{1} f_{2} \frac{3}{4}\left[\left(\cos ^{2} \theta_{\mathrm{s}}+1\right)+\left(\cos ^{2} \theta_{\mathrm{s}}-1\right) \bar{q}\right]$

where $f_{1}$ and $f_{2}$ are given by Eqs. (13) and (15), $\theta_{\mathrm{s}}$ is the scattering angle, and $\bar{q}$ refers to the scattering plane.

\subsection{Photon frequency}

For most of the models discussed here, the O VI emitters are assumed to have zero mean velocity in the binary reference frame. (We neglect the dynamical effects of orbital motion, since orbital velocities are likely to be smaller than wind velocities for all but the shortest-period, slowest-wind systems.)

Scattering events in the expanding wind subsequently introduce frequency shifts through the Doppler effect. If $\hat{\mathbf{p}}$ is the direction of the incident photon packet, $\hat{\mathbf{q}}$ is the direction of the scattered beam, and $\overline{\mathbf{v}}$ is the velocity of the scattering particle, then the frequency of the incident photon packet with respect to the rest frame of the scatterer is

$\nu_{\mathrm{r}}=\nu_{\mathrm{obs}}\left(1-\frac{\hat{\mathbf{p}} \cdot \overline{\mathbf{v}}}{c}\right)$,

where $\nu_{\mathrm{obs}}$ is the frequency of the photon packet in the observer's rest frame. Once the frequency is put in the rest frame of the scatterer, any frequency shift introduced by the Raman scattering may be calculated. The frequency of the Raman-scattered photon packet is given by

$\nu_{\mathrm{Ram}}=\nu_{\mathrm{r}}-\nu_{\mathrm{Ly} \alpha}$ 
where $\nu_{\mathrm{Ly} \alpha}$ is the rest frequency of Ly $\alpha$. The frequency can then be converted back to the observer's rest frame:

$\nu_{\mathrm{obs}}=\nu_{\mathrm{r}}\left(1+\frac{\hat{\mathbf{q}} \cdot \overline{\mathbf{v}}}{c}\right)$.

\subsection{Photospheric scattering}

In a constant-velocity-wind model (for example) there is a finite density at the wind base, and under these circumstances photon packets may reach the red-giant surface without scattering. To treat this case we use a 'core-halo' model of an extended wind and a thin, static photosphere; we treat transfer in the photosphere in a locally planeparallel geometry. The photon packet is incident on the photosphere at an angle $\beta$ to the normal, $\hat{\mathbf{n}}$. The optical depth to scattering, $\tau$, is determined and the vertical optical depth, $\tau_{\mathrm{v}}$, is stored, where

$\tau_{\mathrm{v}}=\tau \cos \beta$

The photon packet is then scattered. If it is Raman scattered, then it goes to the observer in the normal way. If it is Rayleigh scattered, then the new direction and polarization of the packet is found from the phase matrix, and the optical depth to the next scattering determined. The angle of the photon-packet direction with respect to the normal is calculated from

$\cos \beta=\hat{\mathbf{n}} \cdot \hat{\mathbf{u}}$,

where $\hat{\mathbf{u}}$ is the direction of the packet. The vertical optical depth is then recalculated according to

$\tau_{\mathrm{v}}^{\prime}=\tau_{\mathrm{v}}+\tau \cos \beta$

If the new vertical optical depth is negative then the photon has escaped, and the next scattering is performed in the wind.

\section{Implementation}

A computer program, RAMONE, was written to carry out the modelling described in the previous section. An individual model is calculated for a particular viewing angle $\alpha$, where $\alpha$ is the angle between the stellar line of centres and the observer's line of sight, defined such that $\alpha=0^{\circ}$ corresponds to an observer looking along the line of centres through the $\mathrm{O}$ VI source to the giant star, while at $\alpha=180^{\circ}$ the source is occulted by the giant star.

\subsection{The program flow}

The program follows a simple loop structure for each photon packet:

(i) The initial photon position and direction are calculated. (ii) If the photon direction is towards the stellar photosphere the optical depth to the next scattering event is found. If this depth exceeds the scattering depth to the photosphere then the photospheric scattering routine is called; otherwise the photon is scattered in the wind. If the photon direction is away from the stellar disk, then the scattering optical depth to the boundary is found and the photon is forced to scatter before the boundary.

(iii) The nature of the scattering event is determined randomly. The probability of a Raman scattering is given by

$p($ Raman $)=\frac{\sigma_{\text {Ram }}}{\sigma_{\text {Ray }}+\sigma_{\text {Ram }}}$,

so if $U_{\mathrm{R}}<p$ (Raman) then the scattering is a Raman scattering; otherwise it is a Rayleigh scattering.

(iv) If the photon packet is Rayleigh scattered, then its new direction and polarization are obtained from the Rayleigh-scattering phase matrix, its new frequency is determined in the observer's rest frame, and the loop returns to step (ii). If the photon packet is Raman scattered, then it is scattered towards to the observer. If the cool-star disk intervenes then the packet is absorbed; otherwise it is weighted according to the Rayleigh phase matrix and the absorption optical depth to the observer. The packet goes into the accumulating binned output according to its calculated frequency.

Most of the results we present here are based on MonteCarlo simulations of the $\lambda 6825 \AA$ line, with $10^{7}$ input photon packets and errors based on $\sqrt{N}$ statistics.

\subsection{Tests}

Many tests were performed on RAMONE. Since the model is circularly symmetric with respect to the observer for models with $\alpha=0^{\circ}$ and $\alpha=180^{\circ}$, the expected polarization for such models is zero. High signal-to-noise models were run to ensure that the code produced this result, and that the nett value of $U$ was zero.

For models with no absorptive opacity, the fraction of photon packets converted to Raman photons after $n$ or fewer scatterings is

$1-\left(1-\sigma_{\text {Ram }} / \sigma_{\text {Ray }}\right)^{n}$.

Thus for $\sigma_{\text {Ram }} / \sigma_{\text {Ray }} \simeq 0.2(\lambda 1032 \AA)$ or $0.3(\lambda 1038 \AA)$ half the Raman scatterings are preceded by 2 or fewer Rayleigh scatterings. Several models were run to confirm that the code's output was consistent with this.

A grid of models was calculated in order to compare the results of RAMONE with those given by Schmid (1992). The Schmid models have no stellar wind, and RAMONE models were run with a negligible mass-loss rate in order 
to simulate this. Other parameters were adjusted appropriately. A comparison of the line polarizations is shown in Table 1. The qualitative parameter sensitivity of the line polarization is identical for the two models, but RAMONE gives consistently lower Raman-line polarizations, with values approximately $80 \%$ of the Schmid results. Thus the quantitative agreement between the two sets of results is disappointing considering that, in this case, RAMONE was set up to embody essentially identical scattering physics. We have not been able to find a cause for this discrepancy.

Table 1. A comparison between Raman-line polarizations obtained by Schmid (1992; S92) and those obtained in this study (RMN).

\begin{tabular}{cccccccc}
\hline$\kappa_{\text {red }}$ & $\kappa_{\text {UV }}$ & \multicolumn{2}{c}{$q_{\text {sep }}=10$} & \multicolumn{2}{c}{$q_{\text {sep }}=5$} & \multicolumn{2}{c}{$q_{\text {sep }}=2$} \\
\hline & & S92 & RMN & S92 & RMN & S92 & RMN \\
0.0 & 0.0 & 48.8 & 39.2 & 47.7 & 38.4 & 40.2 & 31.5 \\
0.0 & 0.2 & 63.8 & 55.0 & 62.5 & 52.8 & 52.6 & 42.5 \\
0.0 & 1.0 & 82.4 & 76.8 & 80.9 & 74.7 & 67.4 & 59.6 \\
0.2 & 0.0 & 57.1 & 48.9 & 56.0 & 48.5 & 46.8 & 37.8 \\
1.0 & 0.0 & 66.8 & 58.0 & 65.6 & 57.1 & 54.0 & 42.7 \\
\hline
\end{tabular}

More-general tests are difficult to construct; however, many of the numerical results reported in the following sections are in qualitative agreement with the expected behaviour of the models.

\section{A reference model}

The model contains many free parameters, which may be divided into two broad categories: physical (mass-loss rate, absorption cross-sections, etc.), and geometric (e.g., binary phase, binary separation). In Sect. 7 (et seq.) the parameter sensitivity of the Raman-scattered lines is investigated by examining the changes to the Raman-line polarization spectrum resulting from changes in the system parameters. Since there is such a large number of free parameters it is not feasible to construct a complete grid of models, as this would require an impracticable computational expense. Instead we have constructed a benchmark reference model, and have studied the effects of changing the free parameters of that model.

\subsection{Model parameters}

The mass-loss rate of the red-giant star is expected to be an important free parameter. Mass-loss rates for such stars are not generally well known, but Seaquist \& Taylor (1990) used VLA 6-cm radio observations to estimate values for several of the stars surveyed in Paper I. These mass-loss rates are summarized in Table 2; they cluster about a value of order $\sim 10^{-6} M_{\odot} \mathrm{yr}^{-1}$.
Table 2. Radio mass-loss rates for symbiotic stars (from Seaquist \& Taylor 1990)

\begin{tabular}{lcc}
\hline \multicolumn{1}{c}{ Object } & $\begin{array}{c}\text { IR } \\
\text { Type }\end{array}$ & $\begin{array}{c}\dot{M}\left(\times 10 \mathrm{~km} \mathrm{~s}^{-1} / v_{\infty}\right) \\
\left(10^{-6} M_{\odot} \mathrm{yr}^{-1}\right)\end{array}$ \\
\hline RX Pup & D & 1.3 \\
V455 Sco & S & 0.31 \\
RT Ser & S & 0.35 \\
H2-38 & D & 2.3 \\
RR Tel & D & 4.3 \\
He2-106 & D & 2.1 \\
\hline
\end{tabular}

There are, evidently, selection effects in operation, since high mass-loss rates are required before the radio emission, which originates in the volume of the redgiant wind ionized by the hot component, becomes detectable. However, there are other lines of evidence which suggest that the cool components in symbiotic systems undergo relatively high mass-loss rates compared to local bright M giants (Kenyon \& Fernandez-Castro 1987; Kenyon 1988), and are more similar to those of the 'Bulgelike' M giants (Whitelock \& Munari 1992). We adopt $\dot{M}=10^{-6} M_{\odot} \mathrm{yr}^{-1}$ for the reference model.

An explicit value is required for the photospheric radius (and for the wind acceleration) in order to translate the mass-loss rate into a density, through Eq. (1). We adopted a value of $100 R_{\odot}$ for the red-giant radius (e.g., Vogel 1991). For a red-giant mass of $\sim 1 M_{\odot}$, a mass ratio of $\sim 1$, and a binary period of $\sim 1$ year (a 'short-period', Stype system), the binary separation factor, $q_{\mathrm{sep}}\left(=a / R_{\mathrm{g}}\right)$, is then $\sim 5$. The overall scattering geometry is unaffected by simple scalings; that is, similar results will be obtained for models which have the same values of both $q_{\text {sep }}$ and $\dot{M} / R_{\mathrm{g}}$.

The outflow velocities of the red-giant winds are poorly known. A canonical value of $10 \mathrm{~km} \mathrm{~s}^{-1}$, based on CO lines formed at large distances from the photosphere, is frequently adopted in the literature (cf., e.g., Loup et al. 1993), but may not be appropriate for the giants in symbiotic systems. For our reference model we adopt a constant-velocity outflow, with $v(r)=v_{\infty}=50 \mathrm{~km} \mathrm{~s}^{-1}$. The constant-velocity model allows a simple analytical derivation of the path length for given optical depth between any two arbitrary points in the flow (cf. Eq. 11), and the relatively high outflow speed more clearly reveals the velocity structure in the lines. Moreover, there is some evidence in the observations for relatively high-speed winds, as discussed below.

The opacities of the atmospheres of red giants are difficult to calculate, due to the large number of molecular transitions. Recent calculations by Allard \& Hauschildt (personal communication) suggest that the absorption opacity in the red $(\sim 7000 \AA)$ is negligible, and in the UV is 


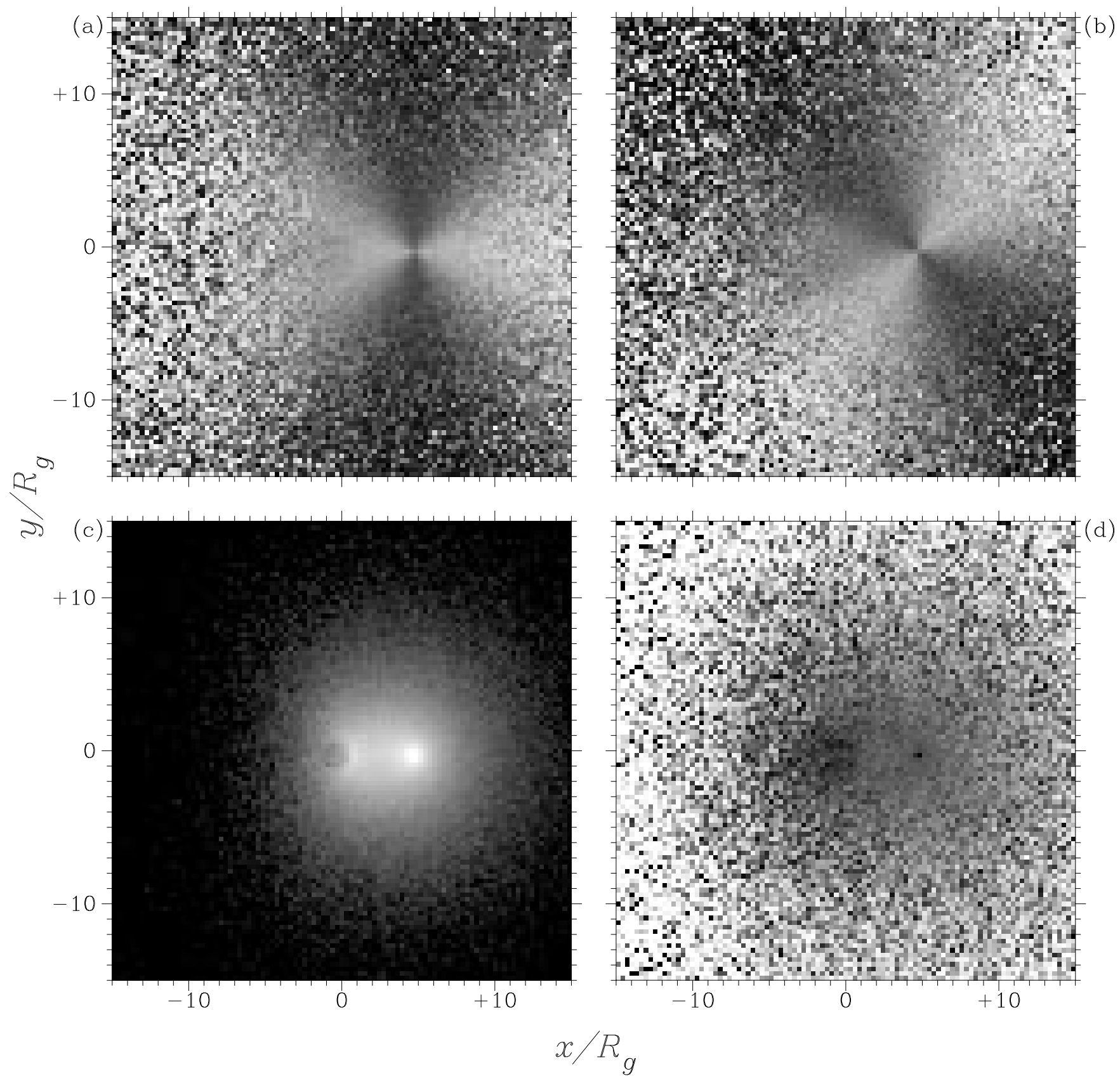

Fig. 2. Results for the reference model ( $\lambda 6825 \AA$ line), viewed at quadrature (Sect. 5$)$ : a), b) are the normalized $Q, U$ images, scaled linearly over the range $-1,+1: \mathbf{c})$, the $I$ image, logarithmically scaled over a range of three decades; and $\mathbf{d}$ ), $I_{\mathrm{p}} / I\left(=\sqrt{U^{2}+Q^{2}}\right)$, scaled linearly over the range $0,+1$. The $\mathrm{O}$ vi source is at $+5,0$, and the cool component (visible as an occulting body in the $I$ image) is at 0,0 
in the range $0-10$ (in units of the scattering cross-section). The opacities in the UV and at the Raman wavelengths are set to zero for the reference model, although the effects of finite absorption at both wavelengths are investigated later.

There is direct observational evidence for fast winds from the hot component in a few symbiotic systems, with outflow speeds of order $10^{3} \mathrm{~km} \mathrm{~s}^{-1}$ (e.g., Nussbaumer et al. 1995). If the O VI source arose in material with such large velocities, any structure in the Raman lines would be completely smeared out (and the lines would be enormously broad). The presence of resolved structure in the Raman lines (Paper I) therefore immediately indicates that most parent photons arise in material which has relatively small, H II-region-like thermal (and 'turbulent') broadening, rather than originating throughout a hot-component wind. This inference is confirmed by recent observations of relatively narrow O VI resonance lines in RR Tel (Espey et al. 1995).

Available observations of the $\mathrm{O}$ VI doublet in symbiotic systems do not resolve the line widths, but highresolution IUE observations of resonance lines of other highly-ionized species, such as Nv, suggest line widths $\simeq 60 \mathrm{~km} \mathrm{~s}^{-1}$ FWHM (e.g., Kindl et al. 1982; Penston et al. 1983). The profiles not unusually show structure which may be of a P-Cygni nature (e.g., Viotti et al. 1983; Michalitsianos et al. 1988) or which may be associated with intrasystem absorption (Shore \& Aufdenberg 1993). For simplicity, we adopt a gaussian distribution for the velocities of particles giving rise to the O VI photons (other frequency distributions may easily be specified), using the GASDEV subroutine of Press et al. (1989) to calculate the deviates. In order to emphasize the structure resulting from velocity fields in the red-giant wind, we take the lines in the reference model to have a relatively low FWHM, $20 \mathrm{~km} \mathrm{~s}^{-1}$, and a mean value of zero (although we explore the consequences of adopting other values).

Table 3. The adopted parameters for the reference model

\begin{tabular}{ll}
\hline \multicolumn{1}{c}{ Parameter } & Value \\
\hline Giant radius, $R_{*}$ & $100 R_{\odot}$ \\
Binary separation factor, $q_{\mathrm{sep}}$ & 5 \\
Mass-loss rate, $\log \left(\dot{M} / M_{\odot} \mathrm{yr}^{-1}\right)$ & -6 \\
Wind speed, $v_{\infty}$ & $50 \mathrm{~km} \mathrm{~s}^{-1}$ \\
FWHM of O vi velocity dist. & $20 \mathrm{~km} \mathrm{~s}^{-1}$ \\
Red opacity, $\kappa_{\text {red }}$ & 0 \\
UV opacity, $\kappa_{\mathrm{UV}}$ & 0 \\
\hline
\end{tabular}

\section{Reference-model results}

In order to examine the distribution of the Raman line formation, the position, frequency, weight, and polariza- tion of each Raman-scattered photon packet were stored for the reference model, viewed at quadrature. The three spatial dimensions were then collapsed to produce twodimensional images of the model outputs, which are shown in Figs. 2, 3, 4. The corresponding spectropolarimetric results are shown in Fig. 5.

The strongest Raman emission in this model originates close to the parent photon source, with the intensity dropping with increasing radial distance simply because of dilution. (This result must be treated with some caution, since the reference model neglects the $\mathrm{H}$ II region which will usually be associated with the hot source; within such a region the number of neutral-hydrogen scatterers will be negligible.) The intensity also falls off rapidly away from the red giant, because of the decreasing density of the wind. The bulk of the Raman scattering therefore occurs between the photon source and the red-giant photosphere, where the density of both $\mathrm{O}^{5+}$ photons and $\mathrm{H}^{0}$ scatterers is high.

Multiple Rayleigh scatterings degrade the directionality of the O VI photon field, and hence the normalized polarized intensity $I_{\mathrm{p}} / I$. Since there is no UV opacity in the reference model, the Raman photons emerge after three Rayleigh scatterings on average; we therefore expect the normalized polarized intensity to be greatest at moderate to large radial distances from the photon source, because the scatterers will see a more nearly radial radiation field (most scatterings occuring close to the source). Figure 2d confirms this expectation, with normalized intensities approaching unity at $\sim 10 R_{\mathrm{g}}\left(\approx 2 q_{\mathrm{sep}}\right)$ from the parent source. Figures $2 \mathrm{a}$ and $\mathrm{b}$ further confirm that the code generates the expected behaviour in $Q$ and $U$.

A second effect of Rayleigh scatterings is broaden the velocity dispersion of the O VI photons. Local scatterings have little effect in this regard, as there is only a small velocity dispersion between the source atoms and scatterers, but more distant scatterings can introduce considerable shifts in the profile; scatterings across opposite hemispheres will introduce shifts corresponding to twice the wind velocity. These shifts are always redshifts, as every point in the wind sees every other point receding from it (the 'expanding universe' paradigm).

\subsection{The $\lambda 6825 \AA$ line}

The spatially-integrated polarization spectrum (Fig. 5) is basically composed of three separate peaks (at 6821, 6831 , and $6836 \AA$ in the $\lambda 6825 \AA$ line), most obvious in the polarized flux, but also identifiable in the intensity spectrum. The polarization in the central peak is orthogonal to the other two. The origin of this characteristic is most easily understood by noting that the polarization-vector directions are centrosymmetric about the parent source (Fig. 4). Because of the cancellation of the $U$ parameter in the spatially integrated flux, the position angle in the models must have a value of $90^{\circ}$ or $0 / 180^{\circ}$ (within the 


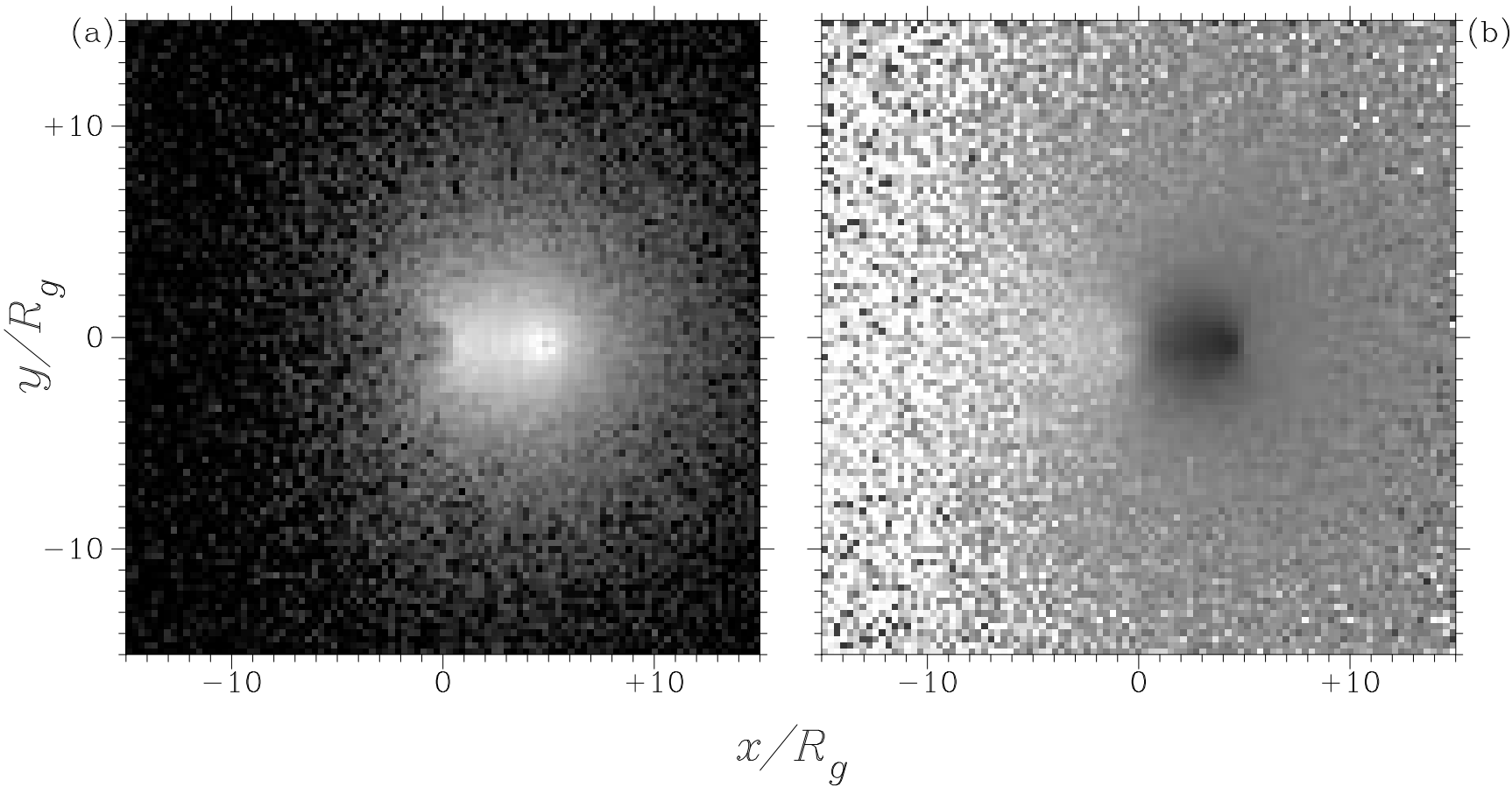

Fig. 3. Results for the reference model ( $\lambda 6825 \AA$ line), viewed at quadrature (Sect. 5). Shown are a) the polarized intensity, $I_{\mathrm{p}}$ (scaled logarithmically over 3 decades), and b) the line-of-sight component of the velocity (scaled linearly over the range -50 to $+150 \mathrm{~km} \mathrm{~s}^{-1}$ )

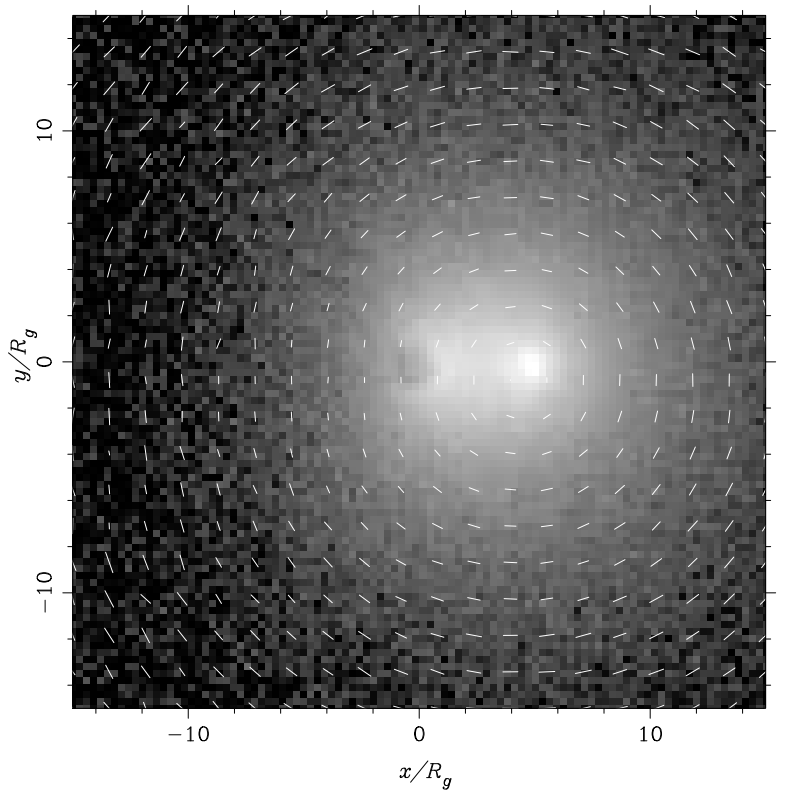

Fig. 4. The polarization of $\lambda 6825 \AA$ Raman photons for the reference model viewed at quadrature, overlaid on a logarithmic intensity scale (scaled over 3 decades). 100\% polarization corresponds to a vector of $1 R_{\mathrm{g}}$ in length errors of the numerical 'noise'). The $90^{\circ}$ features originate 'above' and 'below' the parent-photon source (i.e., along directions perpendicular to both the sight-line and the line-of-centres); thus the spatial origin of a given peak can be deduced from its position-angle dependence.

The bluemost peak is clearly produced in the region between the two stars (the only region of the red-giant wind seen as blue-shifted by the O VI source in the geometry of the reference model). The polarization spectrum is characterized by very high polarization in the blue line wing (as high as 80\%), although there is very little line flux there. The highly polarized blue line-wing is observed in many symbiotics after the removal of continuum flux (cf. Schmid \& Schild 1994; Paper I; Fig. 6).

The central (redshifted) peak has polarization position-angle orthogonally polarized to the bluemost peak, and thus must originate in the two quadrants of the red-giant wind 'above' and 'below' the parent-photon source. The highest-velocity peak is displaced from rest by $\sim 100 \mathrm{~km} \mathrm{~s}^{-1}$ in 'parent-velocity' space. The velocity image (Fig. 3) confirms that this emission arises from scattering across hemispheres, from close to the parent-photon source to the 'back' of the red giant. This third, broad peak is more obvious in the intensity spectrum than the polarized flux (Fig. 5), showing that the 'back' of the wind first sees most photons after they have already undergone multiple scatterings, which produce an effectively spatially 

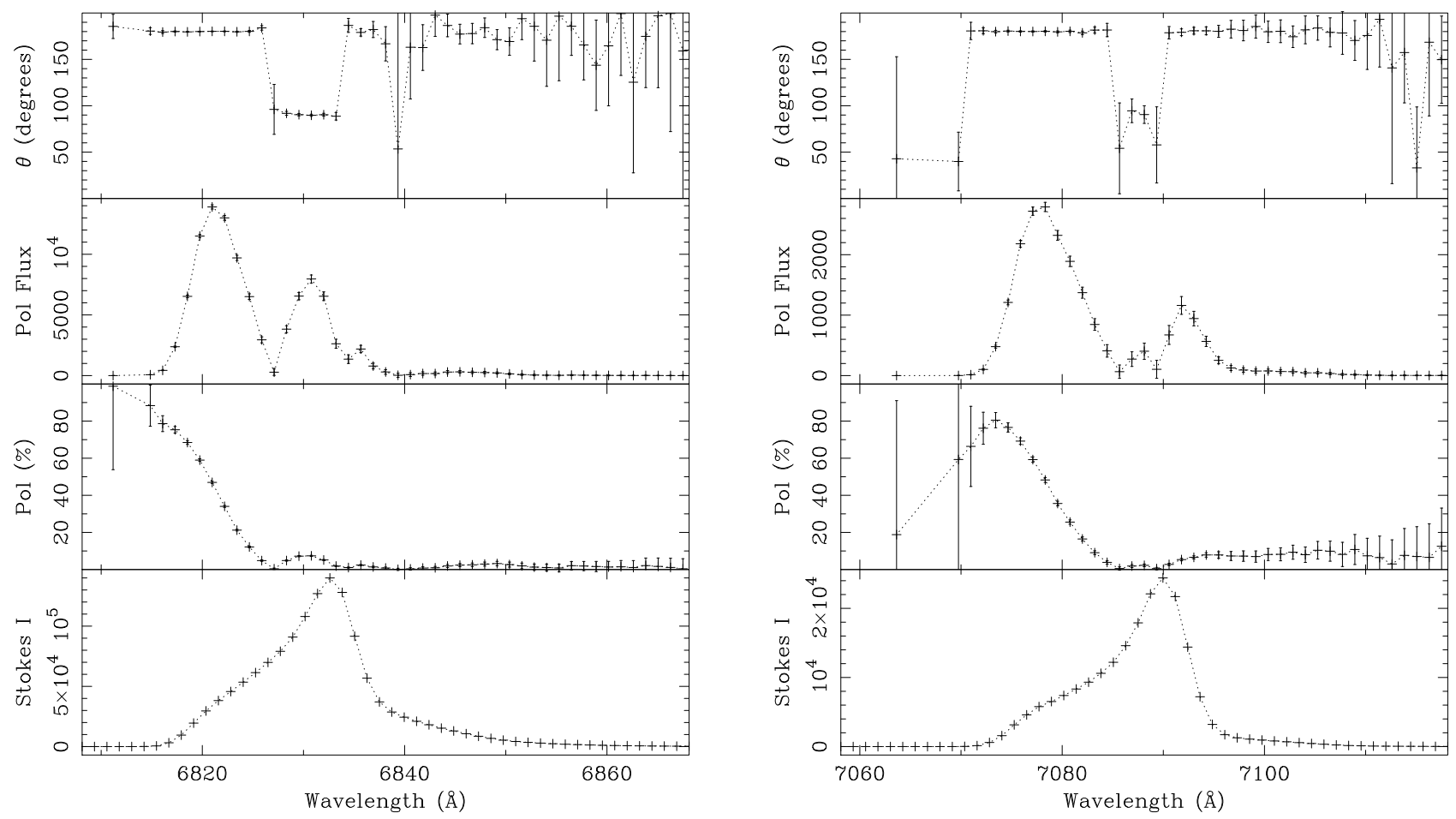

Fig. 5. The polarization spectrum of the reference model (Sect. 5), viewed at quadrature. The error bars are one sigma. Note that the two Raman lines are plotted on different scales

extended source, resulting in reduced polarization (Sect. 11).

Although in our models all photon packets are forced to Raman-scatter, the weighting scheme (Sect. 2.4) ensures that the number ratio of input O vi photons to output Raman photons is correct. In the reference model this ratio is of order 10:1 for $\lambda \lambda 1032: 6085$ - that is, $\sim 90 \%$ of O VI photons escape, in agreement with the observations reported by Espey et al. (1995).

\subsection{The $\lambda$ 7082 A line}

The $\lambda 7082 \AA$ simulation (Fig. 5) shows broadly similar characteristics to the shorter-wavelength Raman line, in agreement with observations (Paper I). The most obvious differences between the lines are the factor $\sim 6.7$ difference in Stokes' $I$ (for an assumed 2:1 photon ratio for $\left.I_{\lambda 1032}: I_{\lambda 1038}\right)$, and the change in relative strength of the central $(\sim \lambda 7090 \AA)$ and redshifted $(\sim \lambda 7095 \AA)$ polarized-intensity peaks.

The observed $I_{\lambda 6825} / I_{\lambda 7082}$ intensity ratios are in the range $\sim 2-10$, averaging $\sim 4-5$ (Allen 1980; Paper I). The reference-model result is slightly larger than this average value, but this mild discrepancy could easily be resolved if $\kappa_{\lambda 1032}<\kappa_{\lambda 1038}$, or if $I_{\lambda 1032}: I_{\lambda 1038}<2: 1$. In our mod- els, the only fixed parameters distinguishing the $\lambda \lambda 6825$ and $7082 \AA$ lines are the Rayleigh- and Raman-scattering cross-sections for the parent OVI photons, which are smaller for the longer-wavelength line. The difference in line intensity in the reference model is therefore directly attributable to the escape of a larger fraction of $\lambda 1038 \AA$ than $\lambda 1032 \AA$ photons (by a factor of $\sim 3$ ).

This also accounts for the differences in line structure: the red-giant wind is quite optically thin perpendicular to the line of centres, so there is little scattered flux from those quadrants, or from the quadrants furthest from the red giant. The blue-shifted polarized flux again arises from the region between the two stars, while the redmost peak arises from multiple scattering, and is slightly more intense (relative to the other two peaks) than the $\lambda 6825$ model because the ratio of Raman:Rayleigh scatterings is slightly higher, meaning that the average Raman photon has undergone fewer scatterings (which reduce the "coherence' of the polarization signal) than in the corresponding $\lambda 6825$ model.

Clearly, observed differences between the $\lambda \lambda 6825$ and $7082 \AA$ lines encode differences in the governing system parameters (e.g., the separate values of $\kappa_{\mathrm{UV}}$ and $\kappa_{\text {red }}$ appropriate to the formation of the two lines). Such differences have important diagnostic potential in 
(a) V455 Sco

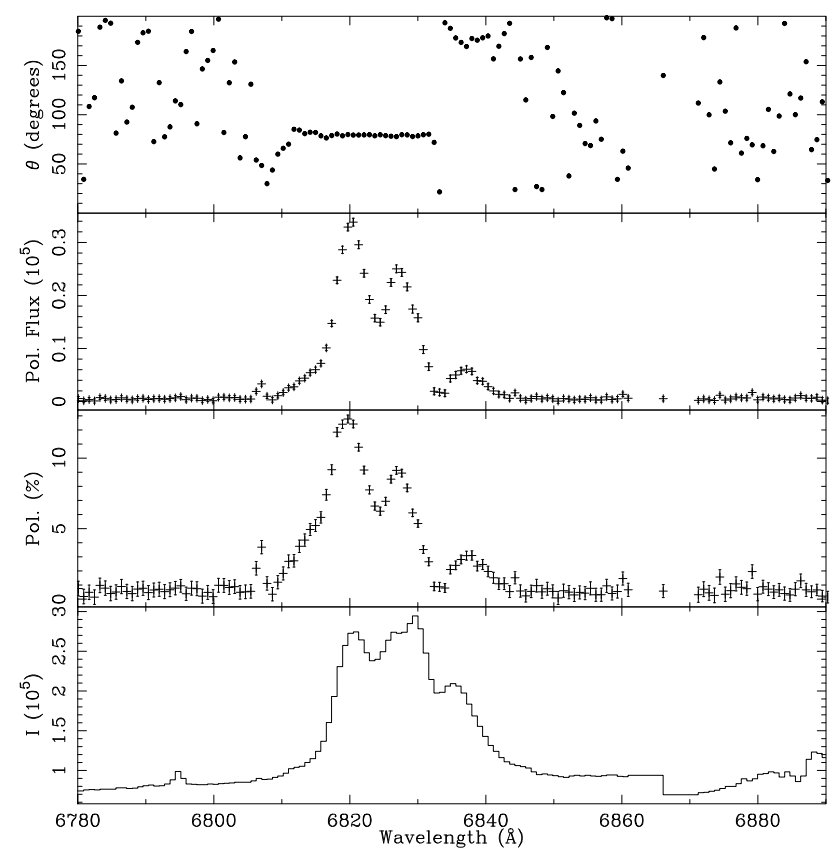

(c) Hen 1242

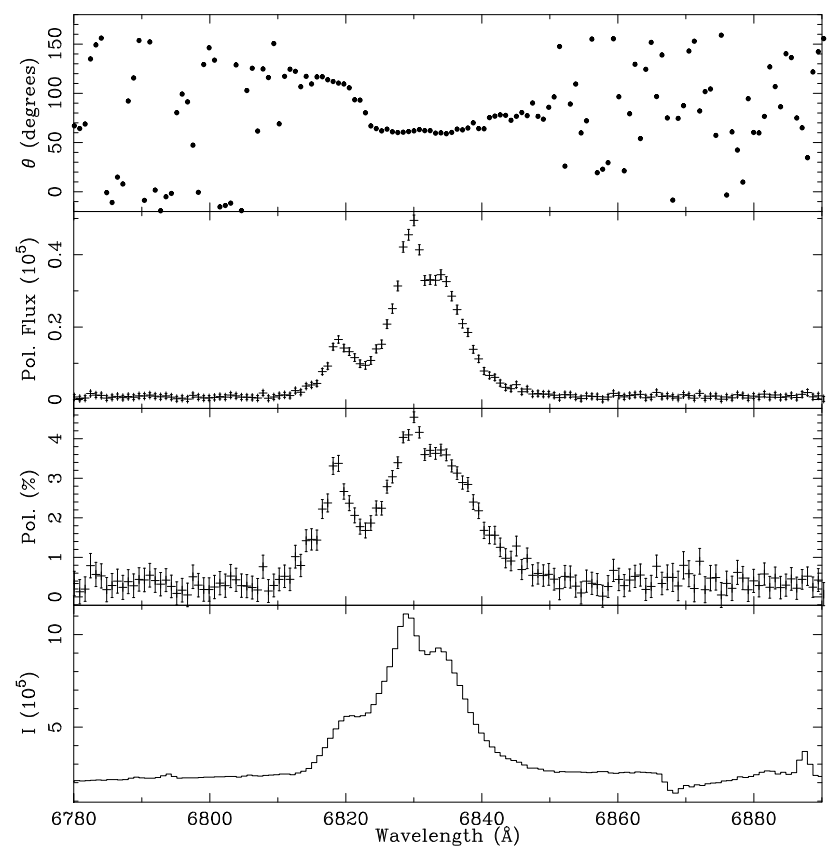

(b) Hen 1092

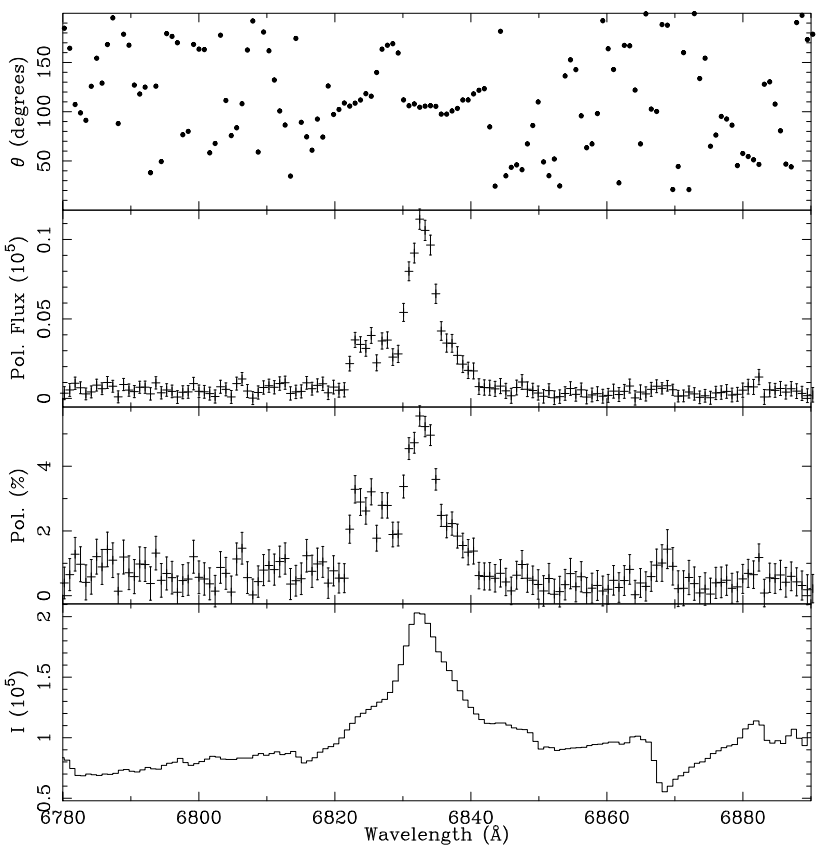

(d) RR Tel

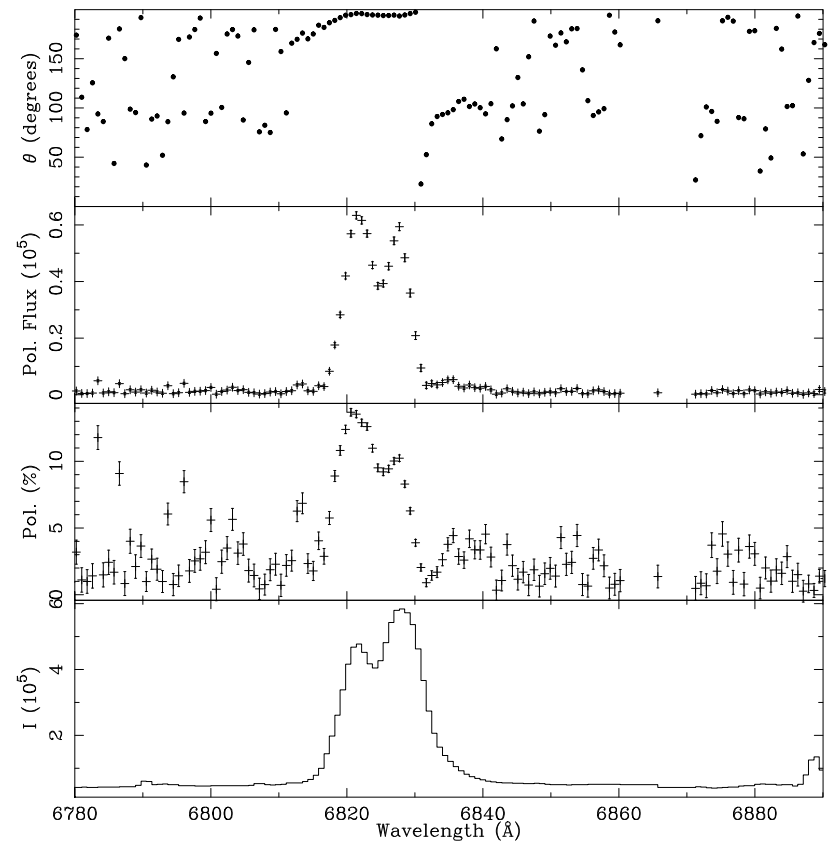

Fig. 6. Observations of the $\lambda 6825 \AA$ line in a) V455 Sco, b) Hen 1092, c) Hen 1242, and d) RR Tel, adapted from Paper I (Harries \& Howarth 1996b). The data shown here have been corrected for the interstellar polarizations derived in Paper I, to facilitate comparison of the percentage polarizations with the models. The polarized-flux and position-angle data in the line are insensitive to such corrections, aside from zero-point shifts 
principle, but the broad similarity of the two lines in the reference model (as well as in observations; Paper I) suggests that the model sensitivity of response of the Raman lines to those parameters can, to first order, be adequately demonstrated by investigating either line separately. Moreover, the $I_{\lambda 6825} / I_{\lambda 7082}$ ratio depends directly on the input $I_{\lambda 1032} / I_{\lambda 1038}$ ratio, which is not well known; a 2:1 ratio is expected under optically-thin conditions, but the resonance doublets of $\mathrm{CIV}, \mathrm{NV}$, and SiIV commonly show smaller ratios in practice (e.g., Shore \& Aufdenberg 1993 and references therein). Henceforth we therefore concentrate on the stronger, and more reliably observed, $\lambda 6825 \AA$ line.

\subsection{Preliminary comparison with observations}

Figure 6 shows polarization data for the S-type systems V455 Sco, Hen 1092, and Hen 1242, and for the D-type system RR Tel. These illustrate part of the diversity of polarimetric behaviour reported in Paper I.

The blueward rise in polarization seen in the model spectra is not apparent in the observations, as dilution by unpolarized continuum flux (which is not included in the model calculation) means that the polarization tends to zero in the line wings. It is possible to subtract off the diluting flux in the data (cf. Fig. 3 in Paper I), but small uncertainties in continuum placement lead to large uncertainties in the polarization, particularly in the line wings. As commented in Paper I, we therefore believe that the polarized flux the more straightforward observable against which to compare the models.

The data for Hen 1092 are broadly consistent with the results from the reference model in that they show a central peak in PA, 'flipped' at $90^{\circ}$ to the adjacent regions, although the relative strengths of the polarizedintensity peaks differ from those in the reference model. The data for Hen 1242, which are of better quality, also clearly show the $90^{\circ}$ flip between the bluemost and central peaks, although the polarized intensity of the bluemost peak is again much less than the reference model predicts. However, in the observations shown in Fig. 6, the central and redmost peaks show the same PA. In both V455 Sco and RR Tel the relative strengths of the intensity and polarized-intensity peaks are in satisfactory agreement with the model, but the central and bluemost peaks show the same $\mathrm{PA}$, with a $90^{\circ}$ degree flip in the redmost peak. Thus these stars show the bluemost peak flipped (Hen 1242), the central peak flipped (Hen 1092), and the redmost peak flipped (V455 Sco, RR Tel) - the last configuration being the commonest, present in at least half the spectra reported in Paper I as showing a 'flip'.

Given the range in observed spectropolarimetric characteristics noted above and reported on further in Paper I, as well as the number of free parameters in the model, it is not unexpected that there should be some disagreements between the data and the reference calculation. In order better to understand these disagreements, we have performed calculations which relax some important basic assumptions of the reference model.

\section{Variants on the reference model}

\subsection{The velocity of the emission source}

The source of the O vi photons is unknown in detail, although it is evidently local to the hot component. The reference model employs a photon source which is static in the binary rest-frame, but if the parent photons are produced in the ionized wind of the cool star then a source which is static in the comoving frame of the wind may be more appropriate. In principle, it may be possible to address this question directly, by studying the orbital velocity dependence of the emission lines and cool-star spectrum, but this will present difficulties in practice. We performed a trial calculation in which the parent photons were assumed to be emitted at zero velocity in the comoving frame of the wind. The results are shown in Fig. 7.

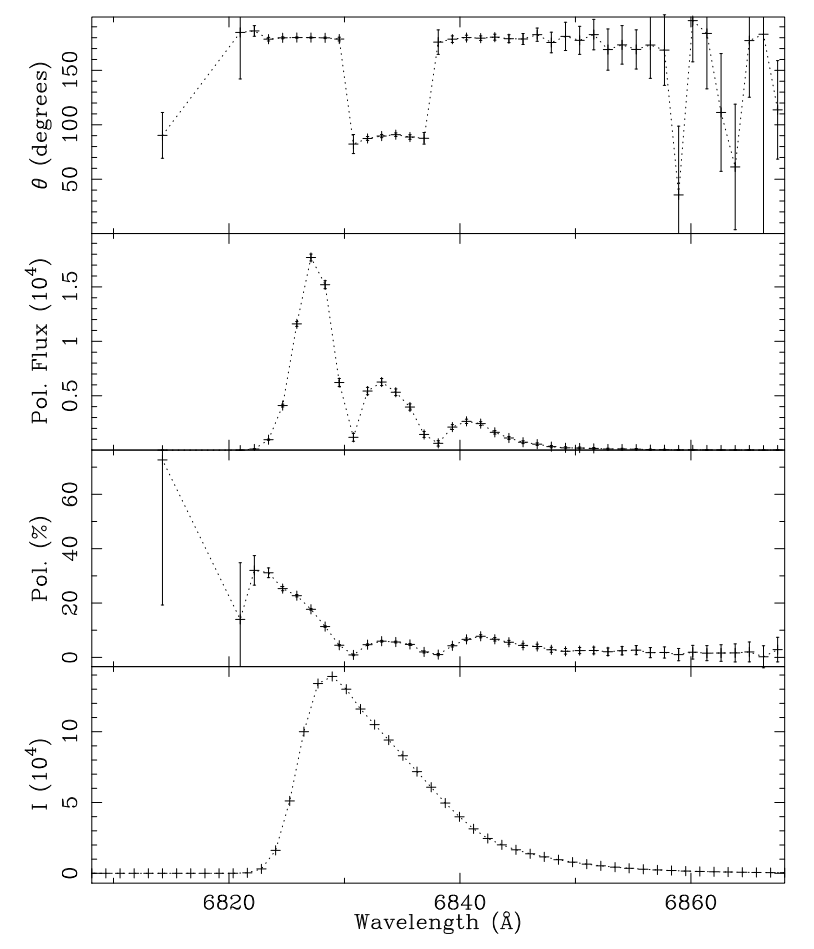

Fig. 7. The polarization spectrum of a model computed with the source emission at zero velocity in the co-moving frame (Sect. 6.1) 
This model shows three polarized-intensity peaks, at about $+20,+60$, and $+110 \mathrm{~km} \mathrm{~s}^{-1}$ (in 'parent velocity' space), with a PA flip for the central peak. This model is therefore qualitatively similar to the reference model, except that essentially all the emission appears redshifted (because the emission source now sees no part of the wind approaching it). The well-observed systems described in Paper I nearly all show a blueshifted peak (cf. Fig. 6). The implication is that our assumption of a photon source which is (on average) static in the binary reference frame is more nearly correct for the majority of real systems than is the assumption of emission from ionized material outflowing from the red giant. There are, however, possible exceptions; a blue-shifted polarized component is undetectable for a few systems (e.g., AS 210, which also shows a constant PA, though not resolved multiple peaks).

The presence of a blue-shifted feature in the Raman lines also constrains any possible shift in the parent-line wavelength. Measurements of emission-line systemic velocities are often difficult to make, but for a few systems observations suggest a red shift in high-excitation lines (e.g., Michalitsianos et al. 1988). Such 'intrinsic' shifts in the parent lines would translate directly into shifts in the Raman-line wavelengths, amplified by a factor $\lambda($ Raman $) / \lambda($ Parent $) \simeq 7$. In our models, a Raman-line redshift results from the expansion of the red-giant wind, and this accounts for the greater part of the observed displacement from the rest wavelengths; the observation of blue-shifted components in the Raman lines shows that any systematic, intrinsic velocity displacement of the O VI lines must be less than the red-giant wind velocity.

\subsection{The ionized region}

Probably the most important simplifying assumption in the reference model is that there is no region of ionized cool-star wind surrounding the hot component. This assumption does not reflect any fundamental limitation of the numerical code, but was adopted simply because to do otherwise complicates the mass-column calculation (Eq. 11) and thereby considerably increases the run time required for a model.

We calculated two models which incorporate extensive ionized zones. In each case, we assumed that the ionized region is completely transparent to all photons. Although this assumption should be relaxed in future work, it is probably not an unreasonable first approximation; in particular, the electron-scattering optical depth along any line through the ionized zone is likely to be $\ll 1$, and so it should not have an important polarization signature. We also assume axial symmetry, which is justified since the recombination timescale is much shorter than the orbital timescale.

In the first model, we approximated the geometry of the ionized zone by

$R_{\mathrm{I}}=1 /(1+2 \cos \Phi)$ where $R_{\mathrm{I}}$ is the distance from the hot component to a point on the ionization front (in units of the binary separation) and $\Phi$ is the angle between that point and the line of centres. In this model, the ionization front is concave to the O VI source. The intensity and velocity images are shown in Fig. 8, and the spectropolarimetry in Fig. 9. Because of the geometry of the ionized zone, there are still extensive scattering regions 'above' and 'below' the O VI source which, as in the reference model, give rise to PAflipped, redshifted, polarized intensity at around $6835 \AA$; thus many characteristics of this model are similar to those of the reference model (including the weak, high-velocity redshifted polarized intensity arising from 'fore-and-aft' scattering).

In order to produce qualitatively different results within the framework of a spherically-symmetric outflow model, it is necessary to incorporate a more extensive ionized zone. To do this, we used

$R_{\mathrm{I}}=1 /(1+2 \cos (2 \Phi))$.

The resulting spectropolarimetry is shown in Fig. 10. Two main intensity peaks remain, corresponding to scatterings on either side of the red giant along (and around) the line of centres. In this model, however, the ionization front is concave to the red-giant component, so that the scattering regions 'above' and 'below' the O VI source have been removed from the model. The PA is therefore constant across the line profile.

These results offer the hope that the PA structure in the Raman lines may prove to be a straightforward, if crude, diagnostic of the extent of the ionized region. In particular, the presence of a PA 'flip' in the Raman lines of many well-observed symbiotic stars (Paper I) suggests that the ionized region may not be very extensive in those systems. In terms of the commonly-adopted parameterization of the extent of the ionized-hydrogen region

$X \propto L_{\mathrm{I}}\left(v_{\infty} / \dot{M}\right)^{2}$

(Seaquist et al. 1984; Nussbaumer \& Vogel 1987; $L_{\mathrm{I}}$ is the luminosity in the ionizing continuum), then the model described by Eq. (34) has $X \simeq 0.5$, and that described by Eq. (35) has $X \simeq 1$. The results presented here therefore imply $X$ values of order unity or less for systems which show a 'flip'. However, Mürset et al. (1991) have estimated $X$ by an independent method for several systems, including three with well-observed Raman lines reported in $\mathrm{Pa}$ per I. All three systems show a 'flip', suggesting small $X$, whereas Mürset et al. report $X$ of order 10 (SY Mus), unity (RR Tel), and $10^{-1}$ (Hen 1092). The origin of this discrepancy must be resolved before either technique can be regarded as yielding secure results, but in particular it will be necessary for the models described here to account correctly for the range of PA structure observed. 


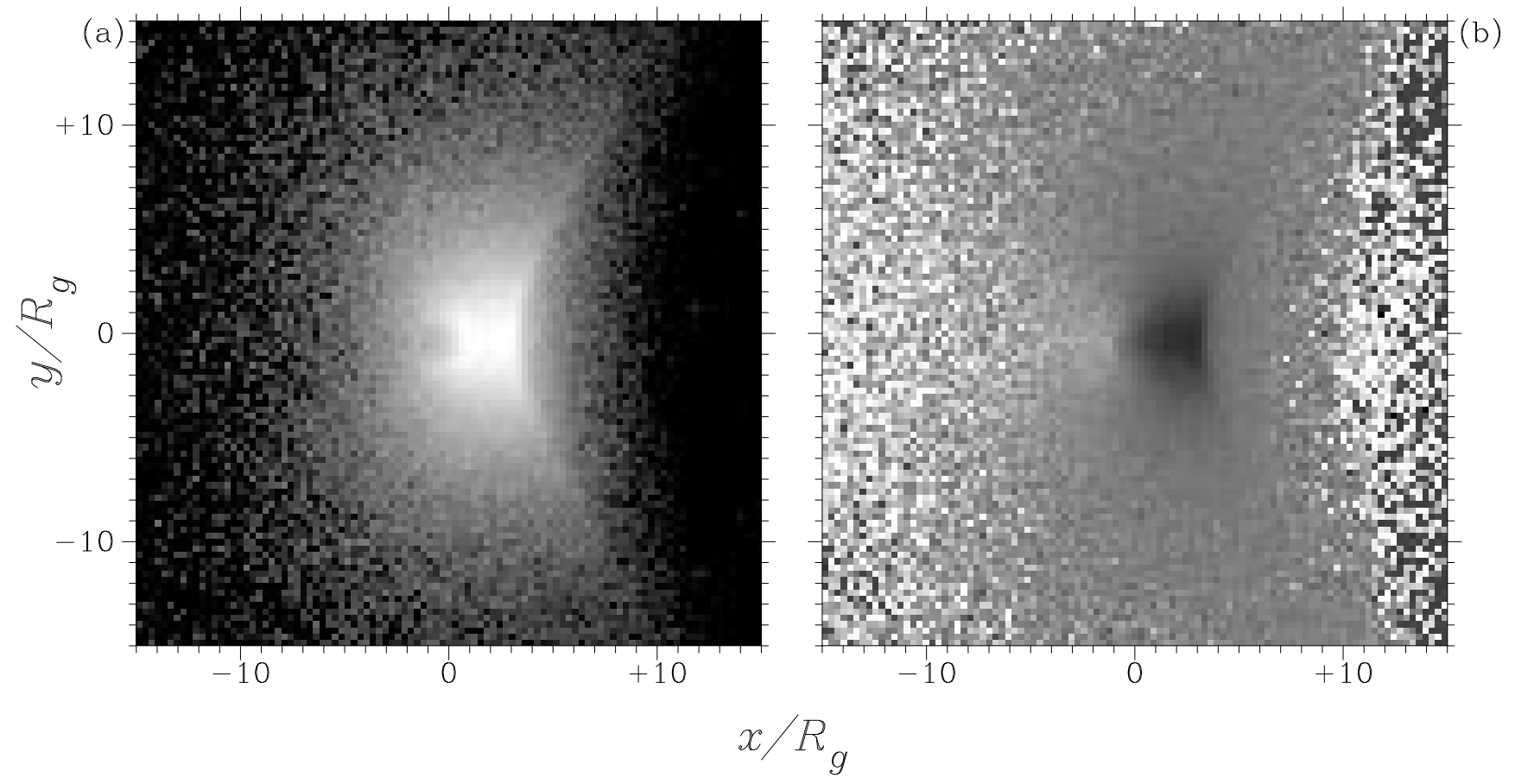

Fig. 8. Results for the reference model, with an ionized region (Sect. 6.2; Eq. (34)) Shown are the intensity, $I$ (scaled logarithmically over 3 decades) and line-of-sight component of the velocity (scaled linearly over the range -50 to $+150 \mathrm{~km} \mathrm{~s}^{-1}$ )

\section{Sensitivity to viewing angle}

\section{1. 'Reflection' models}

The phase dependence of the photospheric scattering was first examined by using the reference-model parameters but with no stellar wind. These models (in common with those reported by Schmid 1992) have all Raman photons produced at the red-giant surface. The polarization,

$P=100 \frac{\left|\sum_{\lambda} Q\right|}{\sum_{\lambda} I}$

and integrated line intensities,

$I_{\mathrm{tot}}=\sum_{\lambda} I$

were measured for models with binary separations of $q_{\text {sep }}=2,5$, and 10, at viewing angles ranging between $0^{\circ}$ and $180^{\circ}$. Plots of the line polarizations and intensities against viewing angle are given in Fig. 11.

As expected, the line intensity is a maximum at $\alpha=$ $0^{\circ}$, the system then being viewed along the line of centres with the O VI source 'in front'. As the viewing angle increases the scattered intensity decreases, principally because of the decreasing area of the scattering surface which is visible. No counts are observed at viewing angles

$\alpha \geq 180^{\circ}-\sin ^{-1}\left(1 / q_{\mathrm{sep}}\right)$ as the scattering surface is then totally occulted by the stellar disk.

The polarization also follows the expected pattern. At $\alpha=0^{\circ}$ the line polarization is zero, to within the model errors. As the viewing angle approaches $\alpha \approx 90^{\circ}$ the polarization reaches a maximum, and then returns to zero. The viewing angle for maximum polarization is noticeably less than $90^{\circ}$ for the $q_{\mathrm{sep}}=2$, a characteristic also noted by Schmid (1992). This because the polarization maximum occurs when the bulk of the photons are scattered through an angle of $90^{\circ}$, and when the source is close to the photosphere this situation occurs at viewing angles of less than $90^{\circ}$.

\subsection{Wind models}

Raman-line profiles were computed for a range of viewing angles $\alpha$ for the reference model and the ionizedwind models. The orbital dependence of the integrated line properties are shown in Fig. 11, and the polarization spectra for the reference model shown in Fig. 12.

The density structure of the wind in these models means that very little scattering occurs in the photosphere, and the bulk of the Raman-line flux is produced within one binary separation of the photon source (see Fig. 4). The phase dependence of the line polarization in the wind models resembles that of the photosphericscattering models, with the polarization maximum 


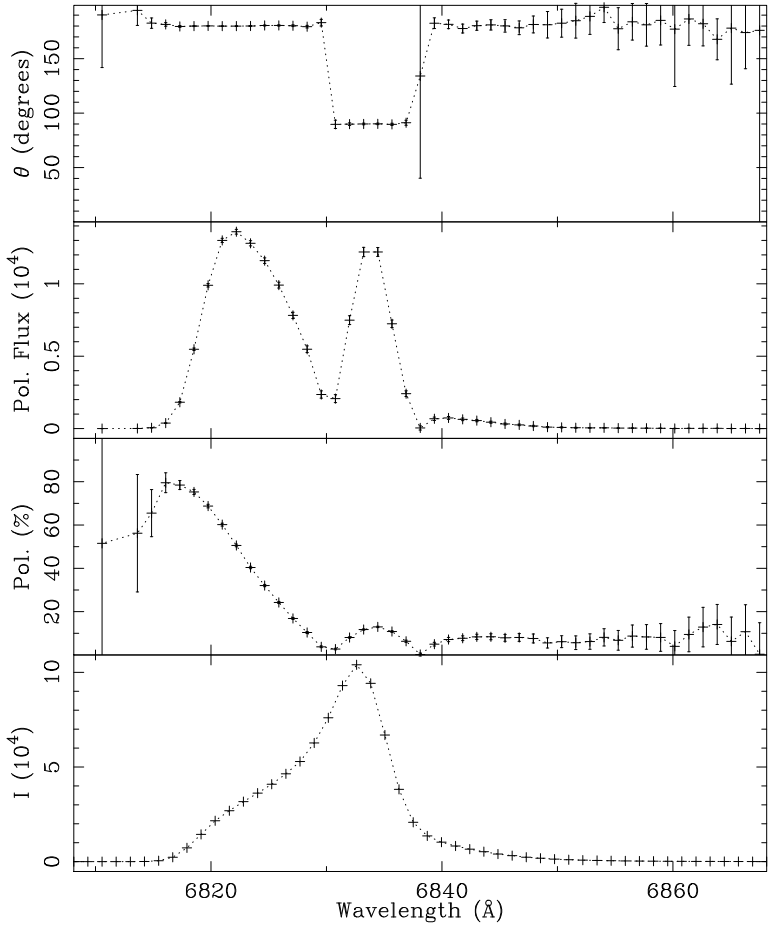

Fig. 9. The polarization spectrum of a model computed with an ionization front which is concave when viewed from the photon source (Sect. 6.2, Eq. (34))

occuring near $\alpha=90^{\circ}$, but the extent of the Ramanscattering region means that the simple reflection-like dependence of the Raman line intensity on phase is lost. In fact, the Raman-line strength and shape is almost constant as a function of phase, although the polarized intensity is more variable.

Schmutz et al. (1994) and Harries \& Howarth (1996a) remark on the near-constancy of the $\lambda 6825$ intensity profile of SY Mus with orbital phase, while Harries \& Howarth note that this is accompanied by significant changes in its polarized-intensity profile. The models shown in Fig. 12 share these characteristics, and offer a simple qualitative explanation for them: the extent of the scattering region is sufficiently large that the occultation effect of the red giant is relatively minor, while the changing parent-scatterer-observer angle directly influences the degree of polarization.

\subsection{Orbital dependence of $P A$}

In the preceding sections, the orbital dependence of the lines was investigated by varying the angle $\alpha$. As already

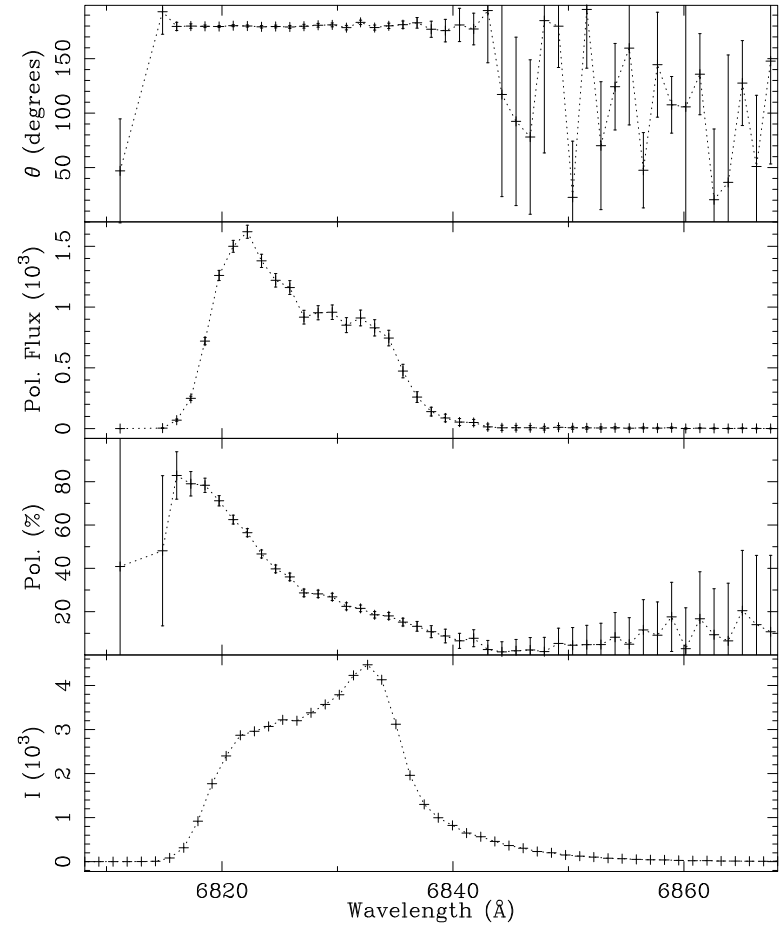

Fig. 10. The polarization spectrum of the model computed with an ionization front which is convex when viewed from the photon source (Sect. 6.2, Eq. (35))

stressed, our models - and any model with 'up-down' symmetry - can only generate position angles of $90^{\circ}$ and $0 / 180^{\circ}$; but, of course, in practice other (orthogonal pairs of) position angles can be generated simply by rotating the observer's reference frame about the line of sight to the system, with $\alpha$ fixed. We make this point explicitly so as to avoid any possible misinterpretation of the discussion in Sects. 7.1, 7.2, but also to emphasize that measurements of polarization position angle provide a straightforward diagnostic of the relative positions of the binary components. The importance of this result is that observed changes in the polarization spectra of symbiotic systems (or other systems in which the source of polarization is scattering of light from one star in the atmosphere of the other) provide a useful diagnostic of orbital parameters, as discussed in more detail by Harries \& Howarth (1996a).

\section{Sensitivity to mass-loss rate}

A change in the mass-loss rate leads to a change in the neutral-hydrogen column seen by the parent photon, and hence the scattering and absorption optical depths. The 
Raman photons will therefore originate in different parts of the wind for different mass-loss rates, and the line profiles will reflect this. Four models were computed in order to investigate this sensitivity, using mass-loss rates of $\log \dot{M}=-5,-6,-7$, and -8 (Fig. 13).

The morphology of the $\log \dot{M}=-6$ (reference-model) polarization profile was discussed earlier. The intensity profile of the $\log \dot{M}=-7$ model shows a two-peaked intensity structure, with the bluewards peak centred at the Raman-line rest wavelength, showing that it is a result of photospheric scattering. The polarized flux is triplepeaked, with all the peaks polarized in the same direction; there is no polarization flip. The three peaks originate in the material between the two stars (approaching the O VI source), in the red-giant photosphere, and in the region 'behind' the red giant (as seen from the hot component). There is, relatively speaking, insufficient material 'above' and 'below' the hot component to introduce significant features at orthogonal polarizations. This interpretation is confirmed by the $\log \dot{M}=-8$ model, which shows a two-peaked intensity profile, with the bluewards peak the strongest. This peak corresponds to photospheric scattering; there is very little scattering in the rarified wind. The polarized flux peak again occurs at the Raman-line rest wavelength, and is a result of photospheric scattering. The very small redward peak is associated with wind scattering in the extended region 'behind' the red giant.

The $\log \dot{M}=-5$ model has a higher intensity than the reference model, and shows a four-peaked structure in polarized flux. The first three peaks (going from blue to red) have the same interpretation as in the reference model, with no significant photospheric scattering. The redmost peak arises because there is sufficient wind density the allow multiple scatterings from 'above' to 'below' the hot component (and vice versa). As expected, therefore, the mass-loss rate (or, more strictly, the density distribution) has a dramatic effect on both the Raman-line intensity and its polarization morphology.

\section{Sensitivity to velocities}

\subsection{The asymptotic wind velocity}

Two test models have been run, with $v_{\infty}=10 \mathrm{~km} \mathrm{~s}^{-1}$ and $v_{\infty}=100 \mathrm{~km} \mathrm{~s}^{-1}$, to check the response of the models to changes in wind velocity. The mass-loss rates were adjusted such that $\dot{M} / v_{\infty}$ - and hence $\rho(r)$ - was constant, so as to isolate velocity effects from density effects. In order to examine the velocity structure of the lines the model profiles, presented in (Fig. 14), have been converted to 'Raman parent' velocity space. The wavelength of the Raman parent photon $\lambda_{\mathrm{p}}$ is given by

$$
\frac{1}{\lambda_{\mathrm{p}}}=\frac{1}{\lambda_{\mathrm{r}}}+\frac{1}{\lambda_{\mathrm{Ly} \alpha}}
$$

where $\lambda_{\mathrm{r}}$ is the Raman-scattered wavelength and $\lambda_{\mathrm{Ly} \alpha}$ is the wavelength of Ly $\alpha$. The Raman parent wavelength may thus be converted to velocity space by using the rest wavelength of the Raman parent line.

The $v_{\infty}=10 \mathrm{~km} \mathrm{~s}^{-1}$ model gives an almost symmetrical profile that is redshifted by about $10 \mathrm{~km} \mathrm{~s}^{-1}$. There is a single peak in the polarized flux spectrum that is blueshifted by approximately $10 \mathrm{~km} \mathrm{~s}^{-1}$ (the reference model has a much broader intensity profile that peaks at approximately $40 \mathrm{~km} \mathrm{~s}^{-1}$, and the polarized-flux peaks occur at $-40 \mathrm{~km} \mathrm{~s}^{-1}$ and $30 \mathrm{~km} \mathrm{~s}^{-1}$ ). The $v_{\infty}=100 \mathrm{~km} \mathrm{~s}^{-1}$ model has a highly asymmetric intensity profile that has a redshifted peak at approximately $90 \mathrm{~km} \mathrm{~s}^{-1}$. The polarized flux spectrum shows three peaks. The blueshifted peak lies at $-80 \mathrm{~km} \mathrm{~s}^{-1}$, the middle peak at $60 \mathrm{~km} \mathrm{~s}^{-1}$ and the redmost peak lies at about $110 \mathrm{~km} \mathrm{~s}^{-1}$.

These results confirm intuitive expectations that the peak-to-peak separations of the polarization profiles should be a function of the local velocity field, and demonstrate that, provided sufficient wind density exists to ensure that scattering occurs over a reasonable volume, the separations of the polarization peaks provide a useful guide to the velocity gradients in the outflow. Because of projection effects, those separations will always be less than the true velocity contrasts, and thus will provide a conservative lower limit if used to estimate wind speeds. The characteristic peak separations of the observations reported in Paper I is $\sim 50 \mathrm{~km} \mathrm{~s}^{-1}$, suggesting that the outflow velocities close to the binary system are rather larger than the canonical $10 \mathrm{~km} \mathrm{~s}^{-1}$.

\subsection{The wind velocity structure}

The reference model employs a constant-velocity wind. Clearly this is an unrealistic assumption and, since the Raman-line velocity structure is most simply explained in terms of scattering in a moving medium, it is of particular interest to examine the effects of different velocities and acceleration laws on the Raman lines. Several models were computed in order to investigate the effects of an accelerating wind on the Raman lines. A velocity law of the form

$v(r)=v_{\infty}\left(1-\left(R_{\mathrm{g}} / r\right)^{2}\right)$

was adopted, solely because, in common with a constantvelocity flow, it has a computationally cheap analytical solution for the mass-column integral over any path in the wind. This velocity law is slightly steeper than those considered by Vogel (1991) when interpreting observations of a Rayleigh-scattering 'eclipse' in EG And. His empirical velocity law has a low, constant velocity out to a few stellar radii, where the wind rapidly accelerates to its terminal speed.

Four models were run, using $\log \dot{M}=-6$ and $\log \dot{M}=$ -7 for binary separations of $q_{\mathrm{sep}}=2$ and $q_{\mathrm{sep}}=5$ (Fig. 15), together with constant-velocity models for comparison. Model (a) shows only minor differences between 


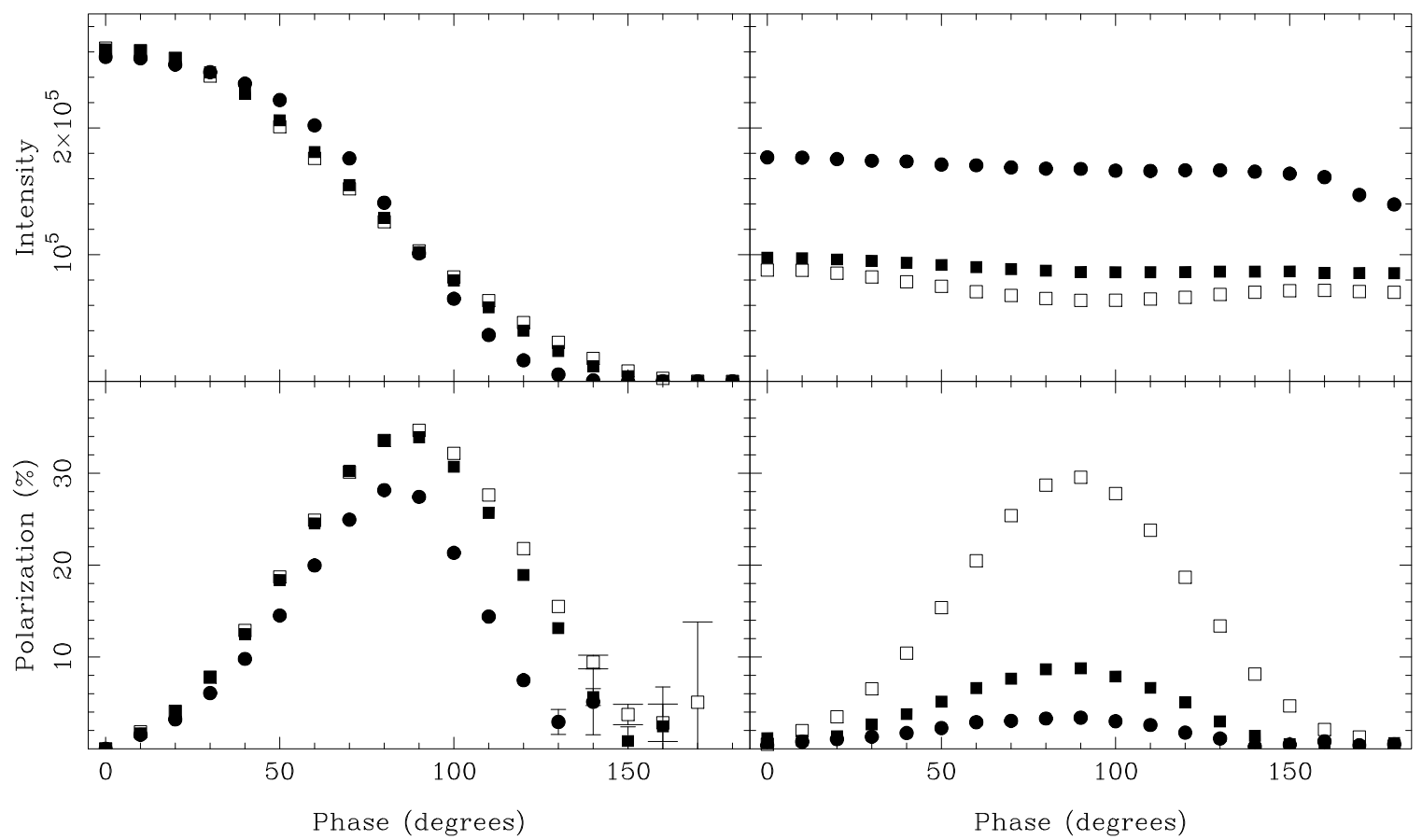

Fig. 11. The phase dependence of scattering models. The left-hand panel shows results for a simple 'reflection' model (Sect. 7.1) with $q_{\mathrm{sep}}=10$ (open squares), $q_{\mathrm{sep}}=5$ (filled squares), and $q_{\mathrm{sep}}=2$ (filled circles); the right-hand panel shows results for the reference model (Sect. 7.2; filled circles) and for models with moderate and extensive ionized zones (Sect. 6.2; filled and open squares, respectively). In each case the top panel shows the line intensity and the bottom panel shows the line polarization. Error bars are one sigma

the Raman line produced by the comparison (reference) model and that produced by the accelerating-wind law. The accelerating-wind models show mild intensity and polarized-flux increases on the blue side of the profile, while the red wings are almost identical. Model (b), with $\log \dot{M}=-7$, shows marked differences in both the intensity and polarized-flux profiles between the accelerating and constant-velocity models. The major changes occur in the blue side of the profile, with the accelerating-wind model displaying a much larger polarized flux in the bluemost peak. The red side of the profile is again almost unchanged, both in total flux and polarized flux.

Model (c), with a mass-loss rate of $\log \dot{M}=-6$ and $q_{\text {sep }}=2$, shows further differences between the two wind structures. The intensity profile of the accelerating-wind model is much stronger, and, although the line polarization is less than the constant-velocity model at the line centre, the polarized flux is greater. The final model, (d), with a lower mass-loss rate and with a small binary separation, gives very different profiles for an accelerating wind. The intensity profile is stronger, with a central peak and red- and blue-shifted shoulders. The polarized-flux profile shows a single strong peak which is blue-shifted with respect to the intensity maximum.
These models demonstrate that the velocity structure of the cool wind can have a strong effect on the Raman lines, both in terms of the intensity profile and the polarization structure. The smallest differences between the constant-velocity wind and the accelerating-wind models occur at the larger binary separations, particularly for winds with high mass-loss rates, because the line formation in the accelerating model is then occurring in regions where the wind is close to its terminal velocity, with little scattering occurring in the steeply accelerating region of the wind.

When significant changes do occur between the constant-velocity and accelerating models, the blue wing shows the most sensitive response. This is because the inter-component region is the scattering volume most affected by the choice of velocity law; the slower the velocity law, the denser the wind in (especially) this region, and the lower the velocity of the scatterers. Thus model (b) shows that the velocity law can be important even in models with lower mass-loss rates and wide binary separations. Clearly, though, the velocity structure becomes most important when the binary separation is small, when the line formation is occurring in a region of the wind with large radial velocity gradients. Model (c) shows that the 
(a)

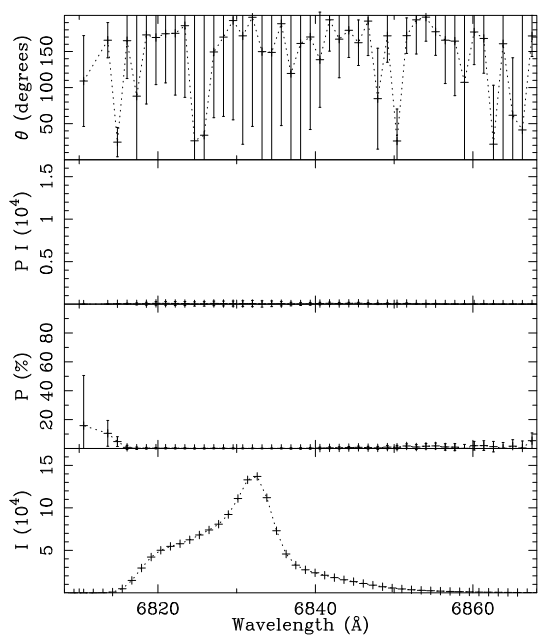

(d)

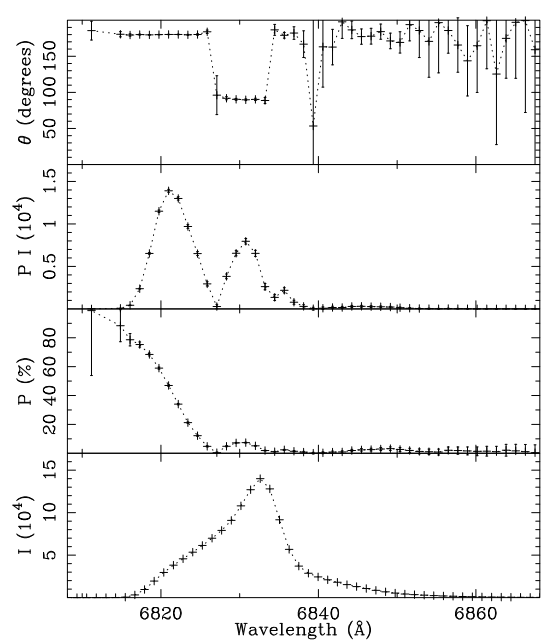

(c)

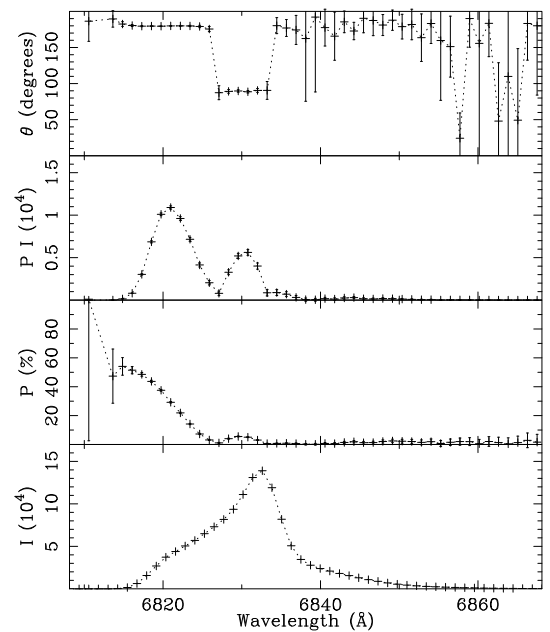

(e)
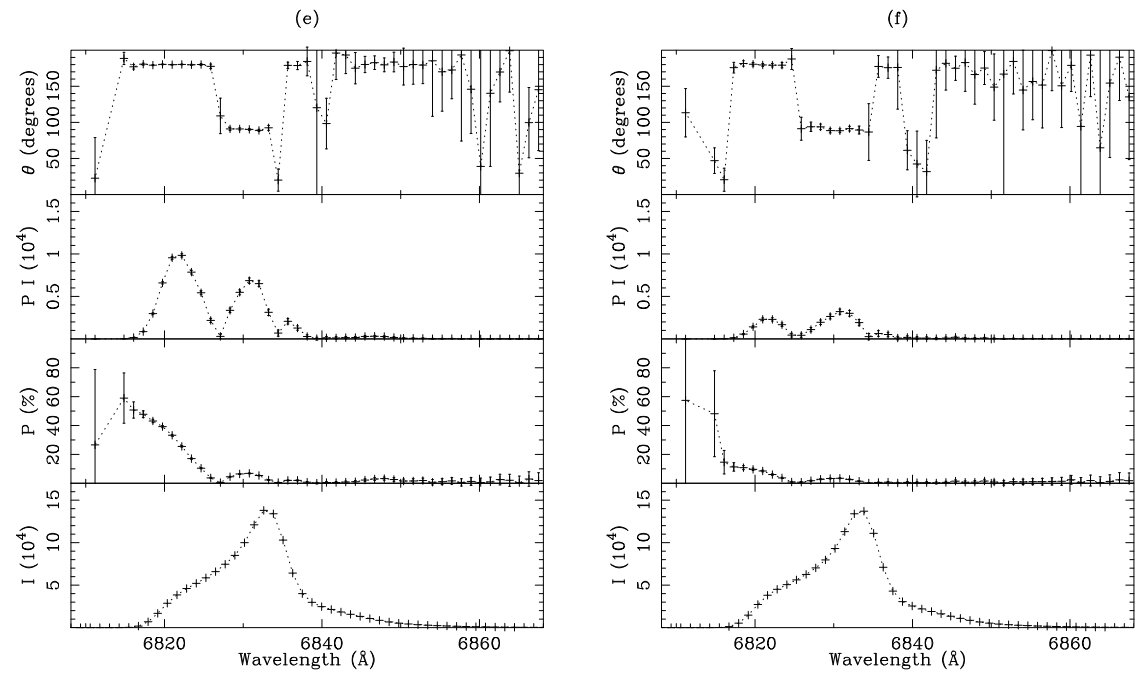

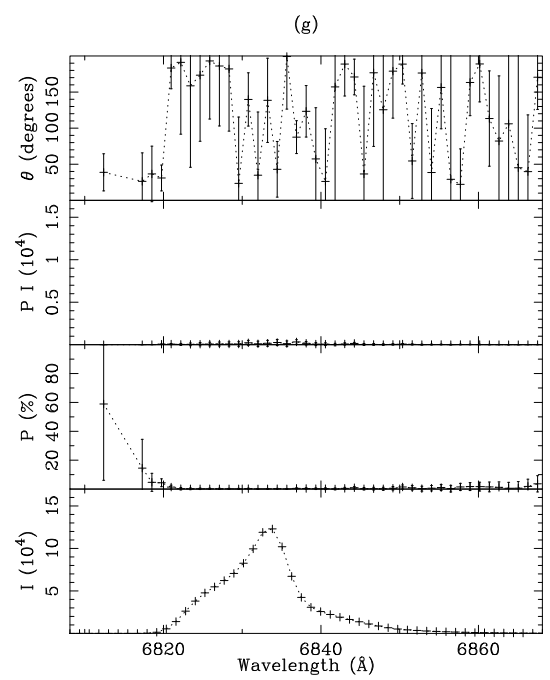

Fig. 12. Raman-line polarization spectra of the reference model with viewing angles of $0^{\circ}-180^{\circ}$ at steps of $30^{\circ} \mathbf{a}-\mathbf{g}$ ) 

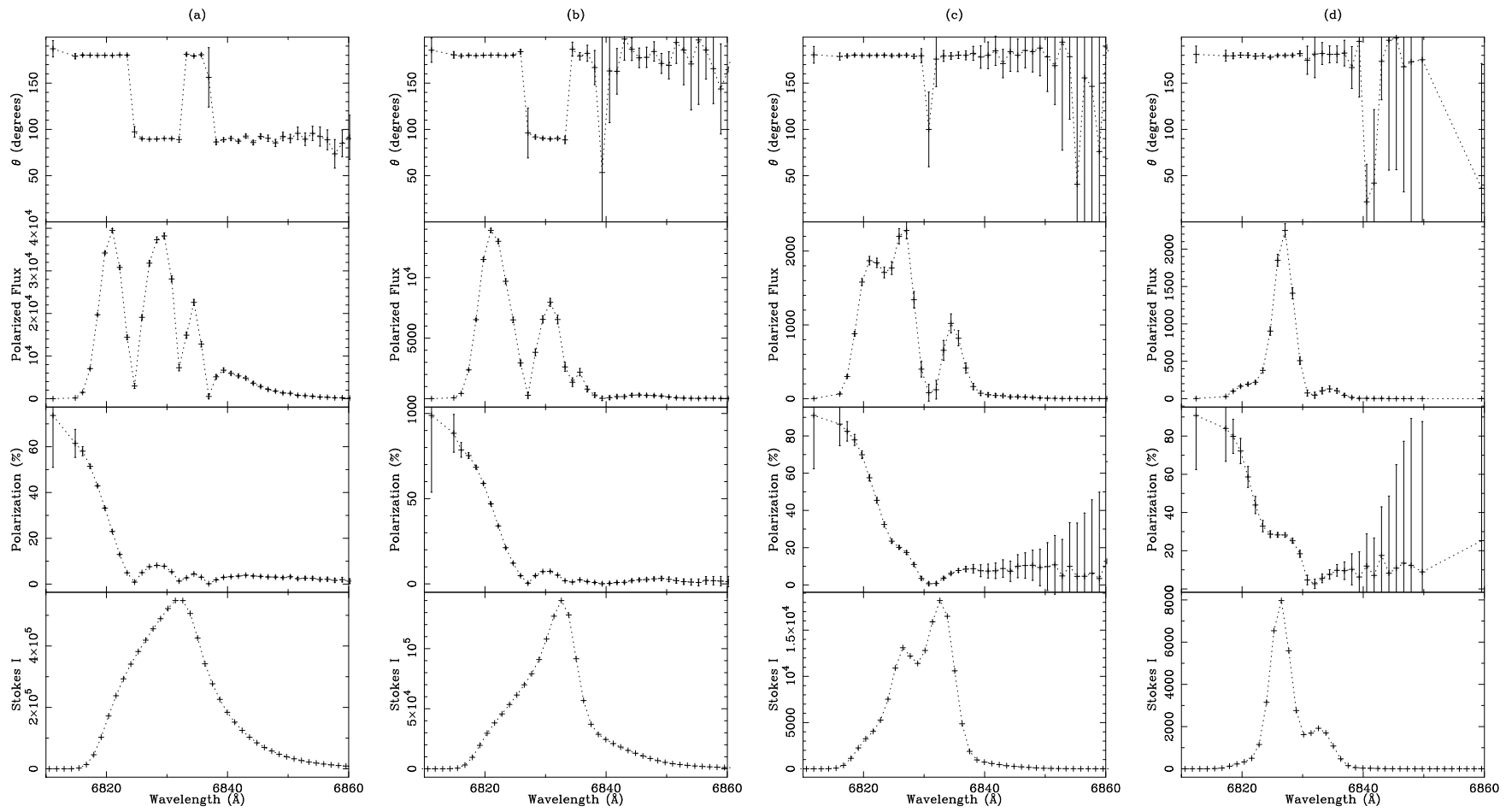

Fig. 13. Raman-line polarization spectra for models viewed at quadrature, with mass-loss rates of a) log $\dot{M}=-5$, b) $\log \dot{M}=-6$, c) $\log \dot{M}=-7$, and d) $\log \dot{M}=-8$ (cf. Sect. 8). Other parameters are those of the reference model

Raman-line intensity is stronger in the accelerating-wind model, mainly because the wind density is much higher at smaller separations than in the constant-velocity model.

Further accelerating-wind models were computed for a small binary separation $\left(q_{\mathrm{sep}}=2\right)$ and low mass-loss rate $(\log \dot{M}=-7)$, and a range of terminal velocities (Fig. 16). These models demonstrate the complex structures that can be obtained when the lines are formed in the accelerating region of the wind, when photons are being scattered in the approaching and receding parts of the wind, and in the photosphere. Model 16(b), in particular, resembles many of the observations presented in Paper I in terms of its PA structure (cf. Fig. 6). The intensity profile shows a strong central peak with blue- and redshifted shoulders, while the polarized-flux spectrum shows a strong blue-shifted peak and two smaller red-shifted ones that are barely resolved. The blue- and red-shifted peaks are perpendicularly polarized. This model has a low massloss rate and a small scattering volume, but because the binary separation is small a PA flip is observed, unlike the $q_{\mathrm{sep}}=5$ model.

\subsection{The $O$ vi line-width}

The widths of the Raman lines are primarily determined by both the velocity field of the red-giant wind and the intrinsic width of the scattered OvI lines. As noted in Sect. 4.1, observations of $\mathrm{N} V$ suggest line widths (FWHM) typically in the range $50-70 \mathrm{~km} \mathrm{~s}^{-1}$; moreover, the line widths increase with increasing ionization potential. We have therefore investigated the effects of adopting broader $\mathrm{O} V \mathrm{VI}$ lines than those of the reference model $\left(\mathrm{FWHM}=20 \mathrm{~km} \mathrm{~s}^{-1}\right)$.

Figure 17a shows the results of a calculation with $\mathrm{FWHM}=100 \mathrm{~km} \mathrm{~s}^{-1}$, and the reference-model red-giant wind velocity. The intensity profile (and, to a lesser extent, the polarized-flux spectrum) is completely unstructured, and has a FWHM of $\sim 1000 \mathrm{~km} \mathrm{~s}^{-1}$. (The 'resolution boosting' of the Raman scattering, by a factor $\lambda($ Raman $) / \lambda$ (Parent), means that the input profile would be broadened to $\sim 670 \mathrm{~km} \mathrm{~s}^{-1}$ even in a static scattering medium.) This is in contrast to the observed lines, which are nearly always highly structured when observed with adequate resolution (Fig. 6; Paper I), and which are rarely as broad as the model shown in Fig. 17.

One might ask if the line width and lack of structure in the intensity profile results from smearing due to 
(a)

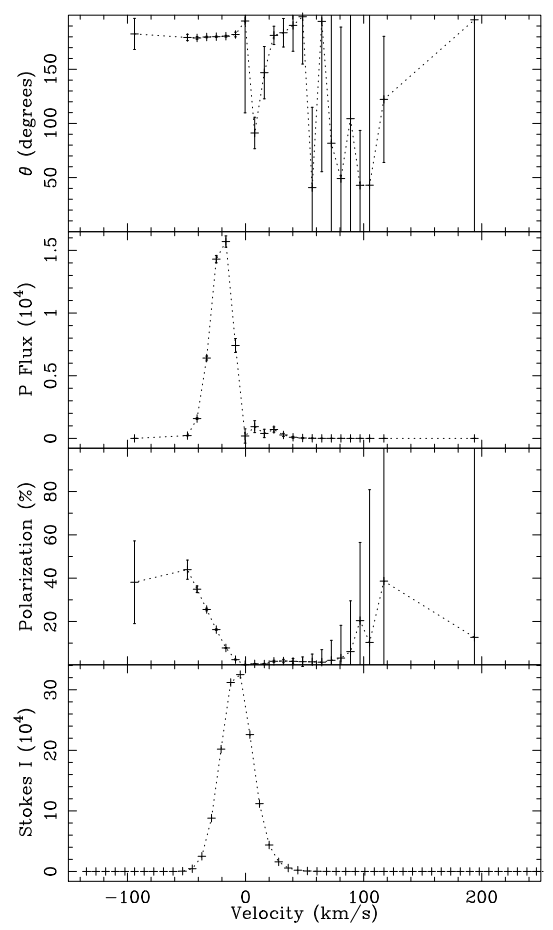

(b)

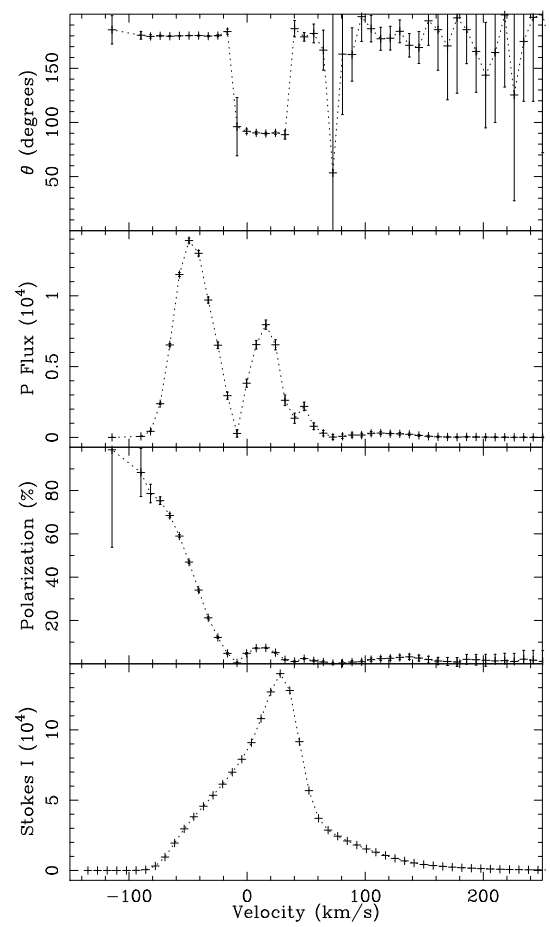

(c)

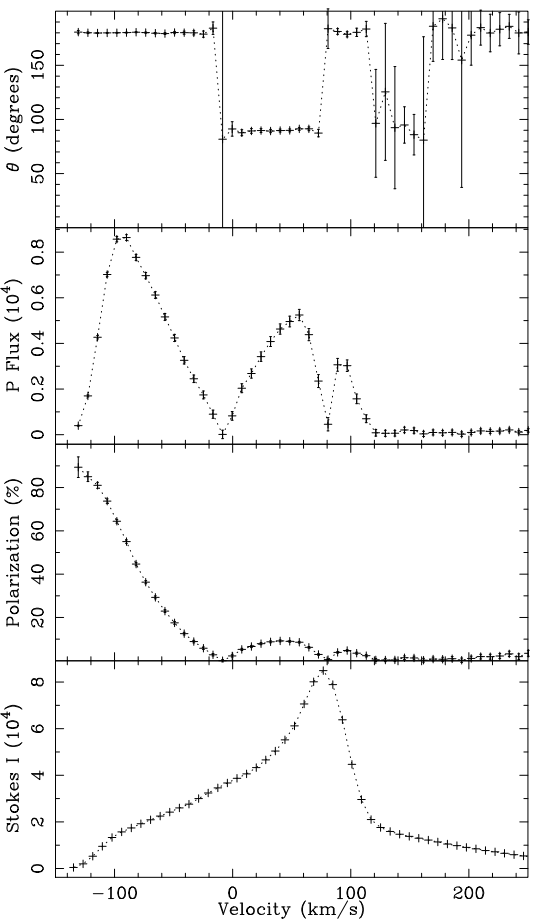

Fig. 14. Raman-line polarization spectra for models with wind velocities of a) $\left.v_{\infty}=10 \mathrm{~km} \mathrm{~s}^{-1}, \mathbf{b}\right) v_{\infty}=50 \mathrm{~km} \mathrm{~s}^{-1}$ and $v_{\infty}=100 \mathrm{~km} \mathrm{~s}^{-1}$, plotted in Raman 'parent' velocity space (cf. Sect. 9.1). The mass-loss rate for models a) and c) were adjusted so that $\dot{M} v_{\infty}$ was equal to that of model b). Other parameters are those of the reference model

the rather large red-giant wind velocity used in the reference model. To address this question we show in Fig. 17b a model calculated with 'canonical' parameters: an O vi line-width of $50 \mathrm{~km} \mathrm{~s}^{-1}$ and a wind velocity of $20 \mathrm{~km} \mathrm{~s}^{-1}$, with $\dot{M} / v_{\infty}$ held at the reference-model value. The intensity profile remains symmetrical and unstructured, with a single polarization peak which primarily arises from scattering close to the line of centres between the stars.

The crucial point of these models is that the velocity width of the $\mathrm{O}$ VI line exceeds the asymptotic velocity of the wind. Hence, the polarization structure can no longer be simply attributed to wind broadening and the effects of spectral smearing must be considered. Figure $17 \mathrm{a}$, for example, has a much reduced red-shifted polarized flux peak. This is because although the mass-loss rate is the same as the reference model (i.e. the number of scatterings above and below the source is the same), the broad $\mathrm{O}$ vi line means that some photons scattered between the source and the red-giant (polarized perpendicularly to the red-shifted peak) have the same wavelength as photons scattered above and below the source, leading to partial cancellation of Stokes $Q$, particularly in the red wing.

To produce a significantly structured polarization spectrum requires an increase in mass-loss rate (i.e., in scattering optical depth). Figure 17c shows a model calculated for the reference-model mass-loss rate. The intensity profile is still unstructured, but the extra scattering optical depth is sufficient to introduce an additional redshifted feature, which, since it is polarized orthogonally to the main peak, clearly originates 'above/below' the O VI source.

These results show that to provide significant structure in the Raman lines (as is nearly always observed) within the framework of the adopted geometry, it is necessary to have a reasonably small O vi line width. The models discussed here therefore provide some justification for the relatively low FWHM adopted in the reference model, and suggest that the scattered component of the $\mathrm{O}$ VI lines may be no more than a few tens of $\mathrm{km} \mathrm{s}^{-1}$ broad.

\section{Sensitivity to absorption opacities}

In our models the UV $\left(\kappa_{\mathrm{UV}}\right)$ and red $\left(\kappa_{\text {red }}\right)$ opacities are quantified in term of the $1032 \AA$ scattering cross-section. These opacities will have a strong effect on the Raman line-formation process. Schmid's (1992) models, and those computed in this study for pure photospheric scattering (Table 1), show identical sensitivity to these parameters. 

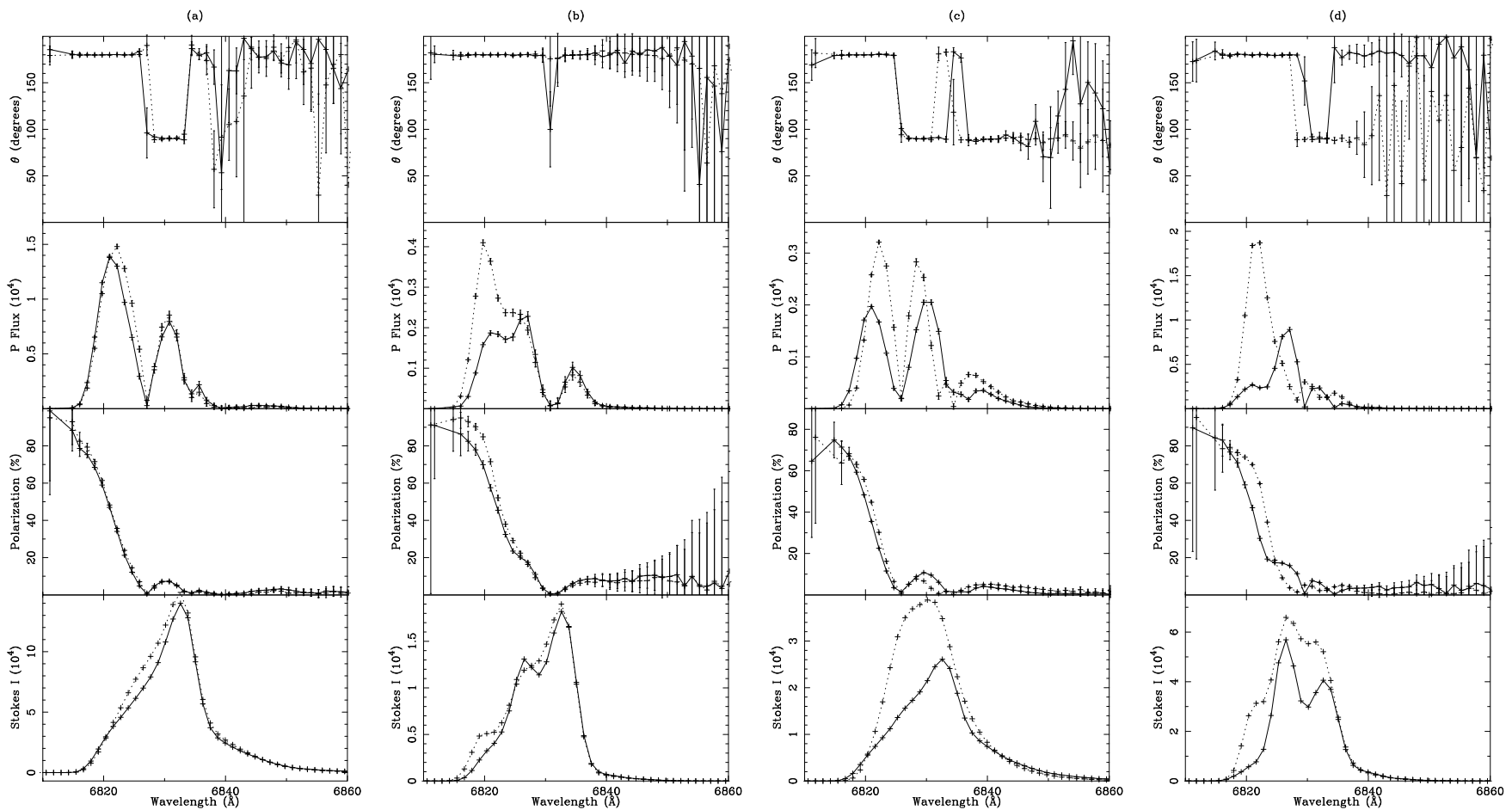

Fig. 15. Raman-line polarization spectra for models computed with a constant-velocity wind (solid line) and with a velocity law $v(r)=v_{\infty}\left(1-\left(R_{\mathrm{g}} / r\right)^{2}\right)$ (dotted line; cf. Sect. 9.2). The parameters are those of the reference model $\left(v_{\infty}=50 \mathrm{~km} \mathrm{~s} \mathrm{~s}^{-1}\right)$ except a) $\log \dot{M}=-6, q_{\mathrm{sep}}=5$ b) $\log \dot{M}=-7, q_{\mathrm{sep}}=5$ c) $\log \dot{M}=-6, q_{\mathrm{sep}}=2$ and d) $\log \dot{M}=-7, q_{\mathrm{sep}}=2$

As expected, an increase in either opacity results in a decrease in the Raman-line strength. However, the opacities have a significant effect on the model line polarizations as well.

Increasing the UV opacity effects only the parent photons. The mean free path of the photons is reduced, and photons may be absorbed before being converted to Raman photons. This results in a bias towards scattered photons originating closer to the parent-photon source than otherwise occurs.

Increasing the red opacity also reduces the observed flux of Raman photons, and although the scattering that causes the polarization of the Raman-line photons occurs in the UV, the red opacity still has an effect on the polarization. Increasing the red opacity in the photosphericscattering models leads to a mild increase in the line polarization because the observed geometrical distribution of the Raman intensity changes.

To quantify these arguments, the reference model was modified by using $\kappa_{\text {red }}=0,1$, and 5 , and $\kappa_{\mathrm{UV}}=0,1$, and 5 (Fig. 18). As expected, an increase in the opacities results in a decrease in the line intensity. The blue side of the profile decreases most rapidly with increasing $\kappa_{\text {red }}$, resulting in a symmetrical, redshifted intensity profile for the $\kappa_{\text {red }}=5$ model. There are also dramatic changes in the polarized-flux profile as the red opacity increases. The bluemost polarized-flux peak observed in the $\kappa_{\text {red }}=0$ model is much reduced in the $\kappa_{\text {red }}=1$ model, and is not produced in the $\kappa_{\text {red }}=5$ profile. The central maximum in the polarization profile increases with increasing $\kappa_{\text {red }}$, in agreement with the pure photospheric-scattering models.

The intensity profiles of the $\kappa_{\mathrm{UV}}$ models retain their general shape with increasing UV opacity, although their intensities decrease. The polarization profiles show a monotonic increase in the central maximum, with the maxima increasing blueshifted. The $\kappa_{\mathrm{UV}}=5$ model shows a redshifted polarization peak that is not present in the other models. As expected, the polarized flux decreases with increasing UV opacity. The wavelength of the position-angle flip is also blueshifted with increasing $\kappa_{\mathrm{UV}}$.

Further models were computed in order to investigate the phase dependence of the polarization spectra for $\kappa_{\text {red }}=1$ and for $\kappa_{\mathrm{UV}}=1$ (Figs. 19 and 20). The integrated line intensities of the models are shown as a function of viewing angle in Fig. 21.

The line profiles of the $\kappa_{\text {red }}=1$ models (Fig. 19) show a significantly different viewing-angle dependence to the reference model. The main differences occur in the blue 
(a)

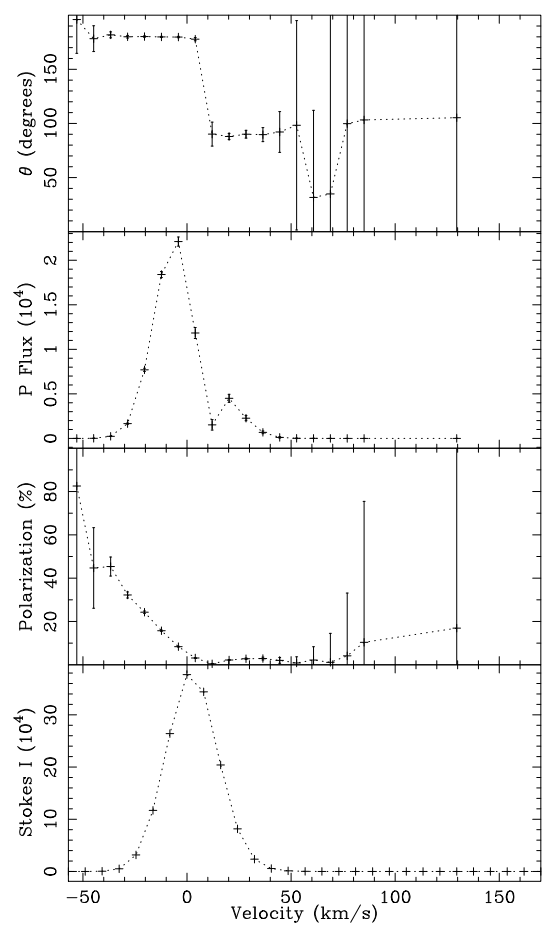

(b)

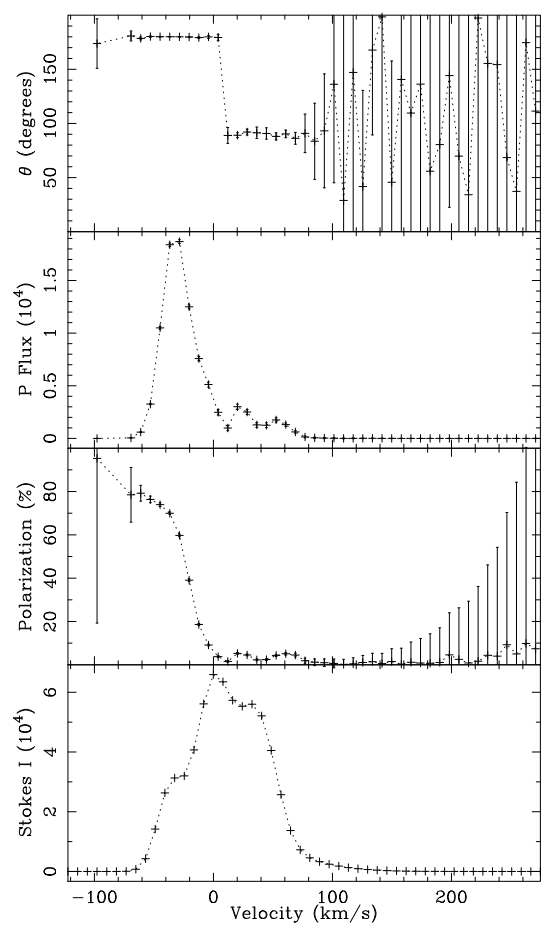

(c)

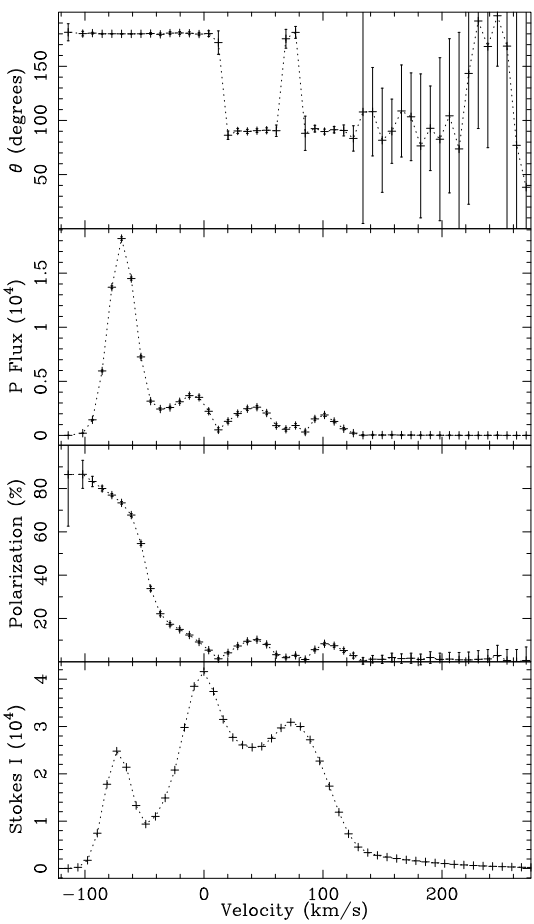

Fig. 16. Raman-line polarization spectra for models with a velocity law $v(r)=v_{\infty}\left(1-\left(R_{\mathrm{g}} / r\right)^{2}\right), q_{\mathrm{sep}}=2$, and log $\dot{M}=-7$ (cf. Sect. 9.2). Other parameters are those of the reference model, except a) $\left.v_{\infty}=10 \mathrm{~km} \mathrm{~s}^{-1}, \mathbf{b}\right) v_{\infty}=50 \mathrm{~km} \mathrm{~s}^{-1}$, and c) $v_{\infty}=100 \mathrm{~km} \mathrm{~s}^{-1}$

wing of the profile, which is much less intense at all phases, but particularly when $\alpha>90^{\circ}$. This is because the blue wing of the line is the result of Raman scattering in the volume of the wind that is approaching the parent photon source. This is also the region with the highest density, and Raman photons that are created in this region have the largest absorption optical depth to the observer. This effect is most clearly seen in the $\alpha=180^{\circ}$ model (Fig. 19g), in which the intensity profile is symmetric and redshifted (since no blue-shifted Raman photons are observed, because of both occultation and absorption in the wind). The integrated line intensity of the $\kappa_{\text {red }}=1$ shows a gradual monotonic decline as $\alpha$ increases and the absorptive opacity of the sightline to the main scattering region increases (see Fig. 21). The integrated polarization shows a peak (in magnitude) at about $\alpha=130^{\circ}$ when the blueshifted polarization peak (which cancels the above/below source scatterings) disappears. Note that $Q$ is negative as the scatterings above and below the source dominate.

The $\kappa_{\mathrm{UV}}=1$ models also show differences when compared with the reference model. The phase dependence of the intensity profile is the same as that of the reference model (Fig. 21), but the polarized-flux profiles are quite different. The blue-shifted polarized-flux peak is ap- proximately the same intensity and the red-shifted peak, over all viewing angles. This is in contrast to the reference model, in which the blue polarized-flux peak is the more intense for all viewing angles $\alpha<150^{\circ}$. The integrated line polarization light-curve is similar to that of the reference model, although the magnitude of the polarization is lower, as the scatterings are occuring closer to the soure, thus reducing the asymmetry of the scattering geometry.

The absorption opacities are important parameters, affecting the strength, intensity profile, and polarization spectra of the Raman-scattered lines. Increasing $\kappa_{\text {red }}$ results in a reduction in the emergent flux from the regions of the wind that have greatest absorption depth to the observer. This generally means that the blue wing of the profile, which results from scattering in the part of the wind that is approaching the photon source, is reduced. The red opacity has less effect on flux that has been Raman scattered in the more tenuous parts of the wind, where the optical depth to the observer is much lower. The UV opacity has a more dramatic effect on the polarization spectra of the Raman lines, in agreement with the pure-photospheric scattering models given by Schmid (1992). 
(a)

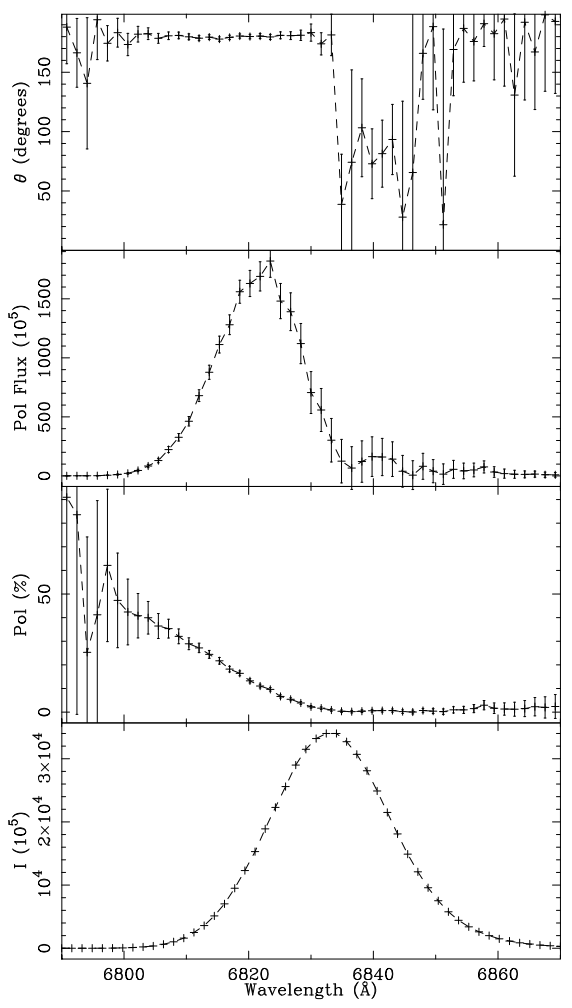

(b)

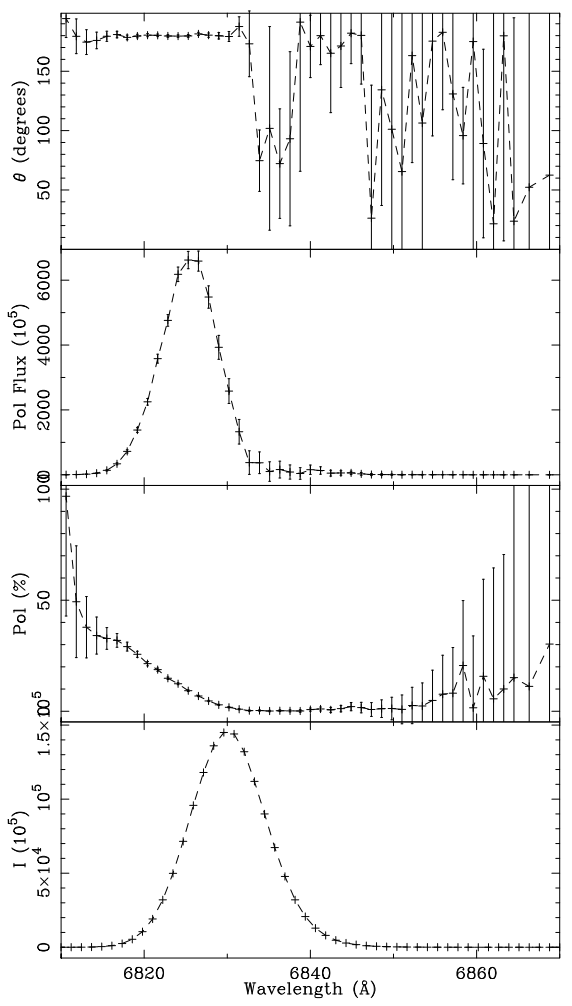

(c)

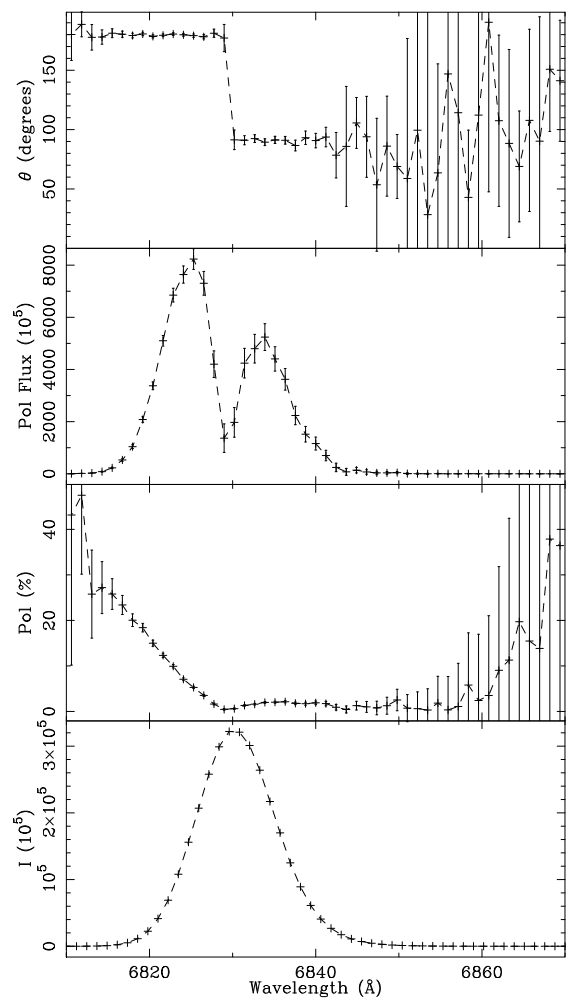

Fig. 17. Raman-line models for $\log \dot{M} /$ parent O vi $\lambda 1032 \AA \mathrm{FWHM} /$ red-giant wind velocity combinations of a) [-6.0/100/50], b) $[-6.3 / 50 / 20]$ and c) $[-6.0 / 50 / 20]$, observed at quadrature (cf. Sect. 9.3)

\section{Sensitivity to photon-source extension}

The models described above were all calculated by assuming a point-like parent photon source. More generally, the O vi photon source may be an extended region, with the size of the region depending on the characteristic temperature and luminosity of the ionizing radiation field and the density structure of the nebula surrounding the hot component. We have carried out calculations for emission from an optically thin, homogeneous sphere of radius $R_{\mathrm{s}}$. This may not be a physically realistic geometry in general, but was chosen for simplicity (other geometries are easily treated).

The normalized probability of photon emission within radius $r$ is

$p(r)=\frac{\int_{0}^{r} 4 \pi r^{2} \mathrm{~d} r}{\frac{4}{3} \pi R_{\mathrm{s}}^{3}}=U_{\mathrm{R}}$

where $U_{\mathrm{R}}$ is a uniform random deviate in the range $0-1$. The radial offset of a parent photon packet from the (point) hot source is then

$r=U_{\mathrm{R}}^{\frac{1}{3}} R_{\mathrm{s}}$ and the initial location in the Cartesian frame is

$$
\begin{aligned}
& x_{0}=q_{\mathrm{sep}} R_{\mathrm{g}}+r u_{0} \\
& y_{0}=r v_{0} \\
& z_{0}=r w_{0}
\end{aligned}
$$

where the direction cosines of the position vector of the initial photon packet direction are obtained from two uniform random deviates:

$w_{0}=2 U_{\mathrm{R}}(1)-1$

$u_{0}=t_{0} \cos \phi$

$v_{0}=t_{0} \sin \phi$

with $t_{0}=\sqrt{1-w_{0}^{2}}$ and $\phi=2 \pi U_{\mathrm{R}}(2)$.

The reference model was recalculated with an extended source $\left(R_{\mathrm{s}}=4 R_{\mathrm{g}}\right)$; in addition, models were calculated with and without an extended source for $\log \dot{M}=-8$ and for $\kappa_{\mathrm{UV}}=1$. The polarization spectra of these models are plotted in Fig. 22. 
(a)

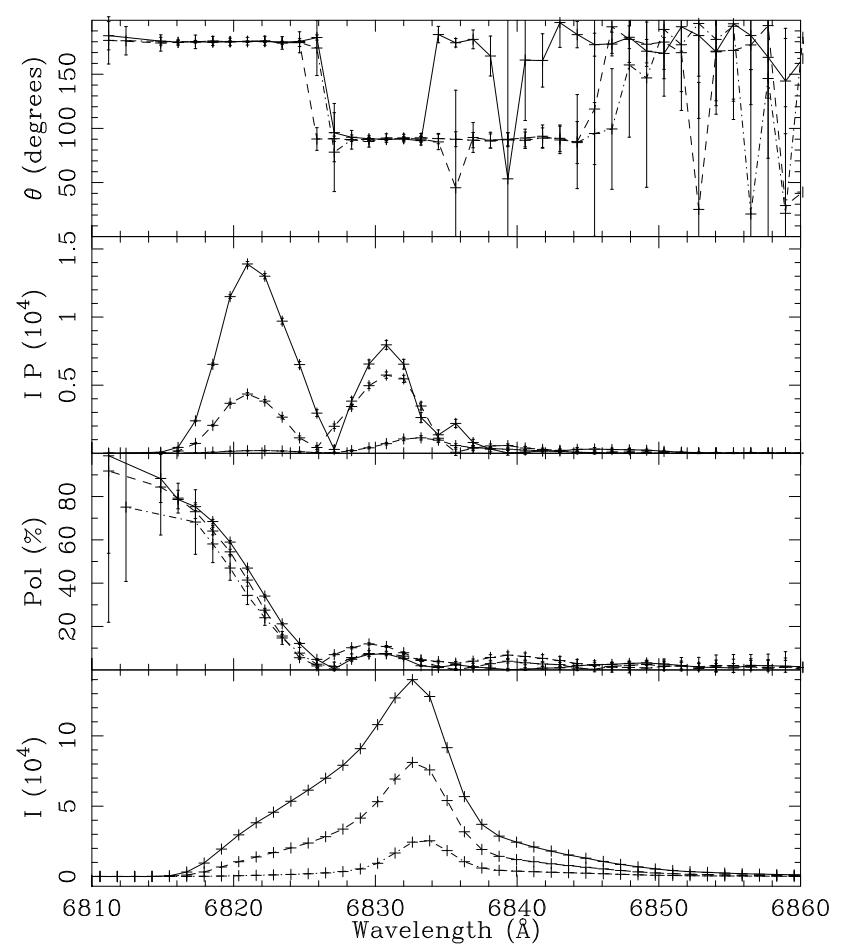

(b)

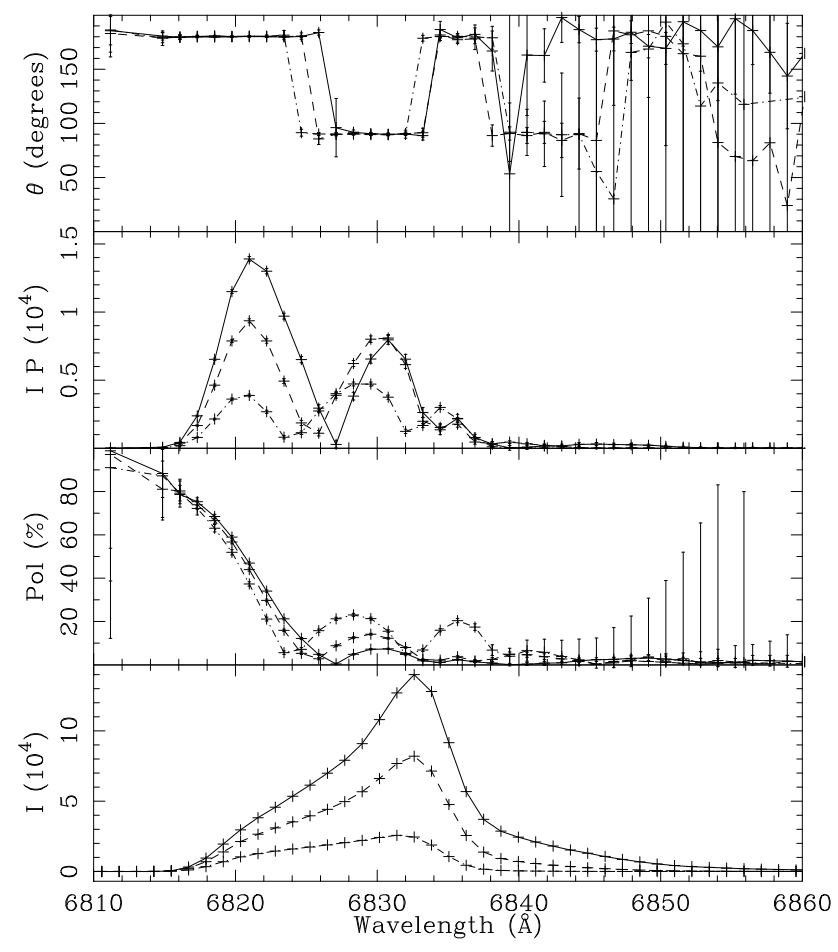

Fig. 18. Raman-line models for different absorption opacities (cf. Sect. 10). Model a) shows that reference model with $\kappa_{\text {red }}=0$ ( solid line), $\kappa_{\text {red }}=1$ (dashed line) and $\kappa_{\text {red }}=5$ (dot-dashed line). Model b) shows the reference model with $\kappa_{\mathrm{UV}}=0$ (solid line $), \kappa_{\mathrm{UV}}=1$ (dashed line) and $\kappa_{\mathrm{UV}}=5$ (dot-dashed line $)$

The polarization spectra of the extended-source models generally have a similar shape to their point-source counterparts, but a much reduced magnitude. As a result, the polarized-flux spectra of the extended-source models are simply lower-intensity versions of the point-source models. This reduction in polarization is straightforwardly understood. The radiation field of an extended source is less 'forward peaked' than in the point-source case; that is, photons incident on the first scatterer will come from a finite cone angle. Thus the nett polarization resulting from the first scattering will be much reduced. Subsequent Rayleigh scattering will dilute the polarization signal from the first scattering, but the resulting polarization will still be reduced.

\section{Discussion}

The numerical models described above are successful in reproducing many of the observed characteristics of the Raman lines in symbiotic systems, and in helping to understand how the spectra respond to changes in the model parameters; there are also several points on which they fail, suggesting ways in which they may be extended and improved.

\subsection{Successes}

Table 12.1 summarizes two basic quantities derived from selected models: the 'polarization efficiency' of the line,

$\eta=\int \sqrt{Q_{\lambda}^{2}+U_{\lambda}^{2}} \mathrm{~d} \lambda / \int I_{\lambda} \mathrm{d} \lambda$

and its first moment (or 'mean wavelength')

$\bar{\lambda}=\int I_{\lambda} \lambda \mathrm{d} \lambda / \int I_{\lambda} \mathrm{d} \lambda$

expressed as a velocity. By comparing the data in Table 12.1 with the corresponding observational data presented in Paper I, we note that (i) the overall redshifts found in the observational data are reproduced by the models (which give reasonable quantitative agreement with the mean observed value of $265 \mathrm{~km} \mathrm{~s}^{-1}$ ), and (ii) that the observed values of $\eta(0.017$ to 0.27 ; mean 0.074$)$ are encompassed by the range found in the models. In addition, the reference model returns values for photon number ratios, $N_{\lambda 6825} / N_{\lambda 7082}\left(\simeq I_{\lambda 6825} / I_{\lambda 7082}\right) \simeq 6$ and $N_{\lambda 1032} / N_{\lambda 6825} \simeq 10$, which are in reasonable agreement with observations (average $N_{\lambda 6825} / N_{\lambda 7082} \simeq 4-5$, Allen 
(a)

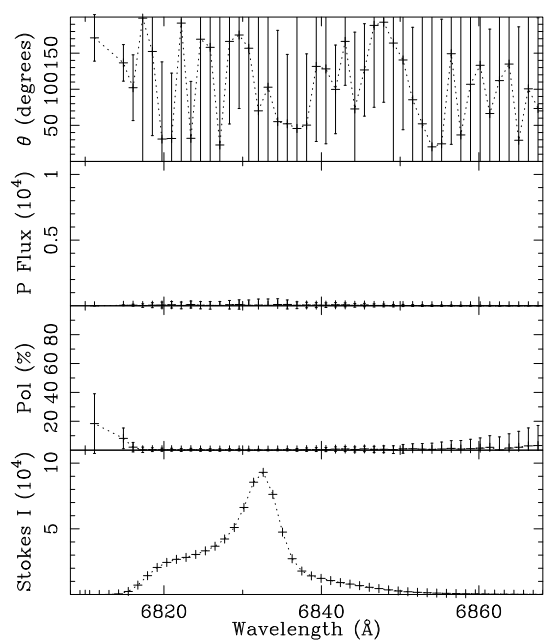

(d)

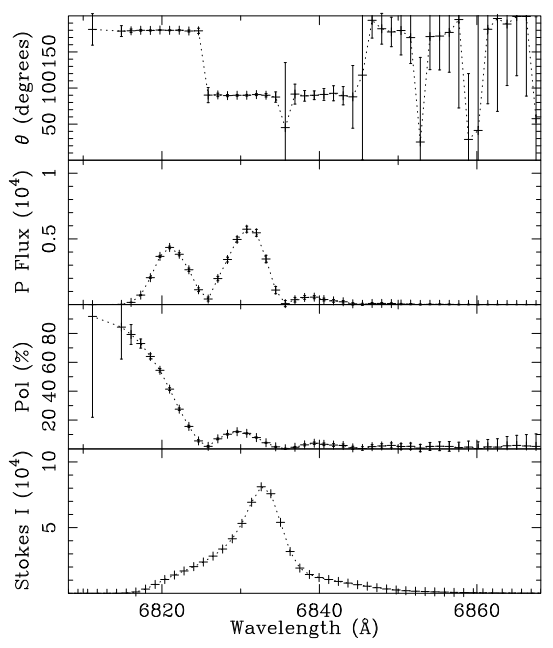

(c)

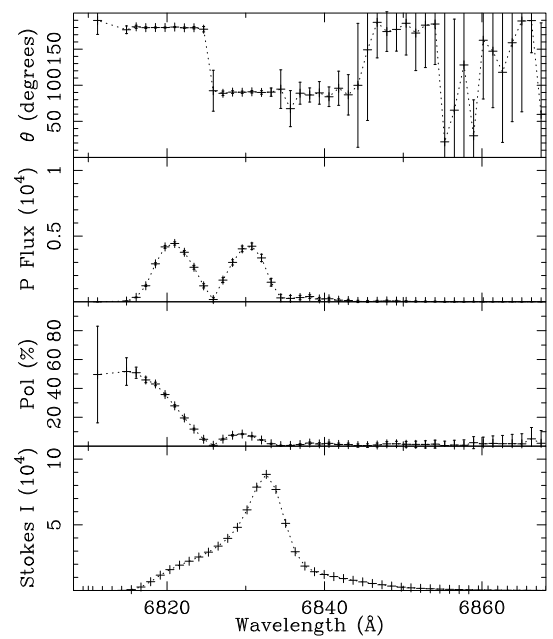

(e)

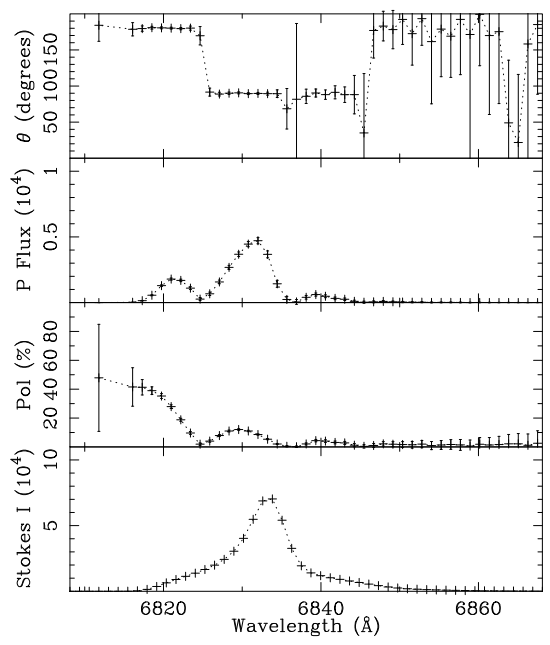

(g)

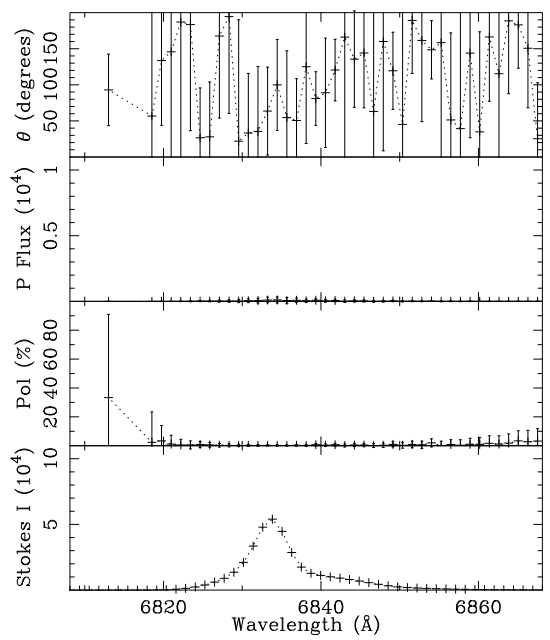

(f)

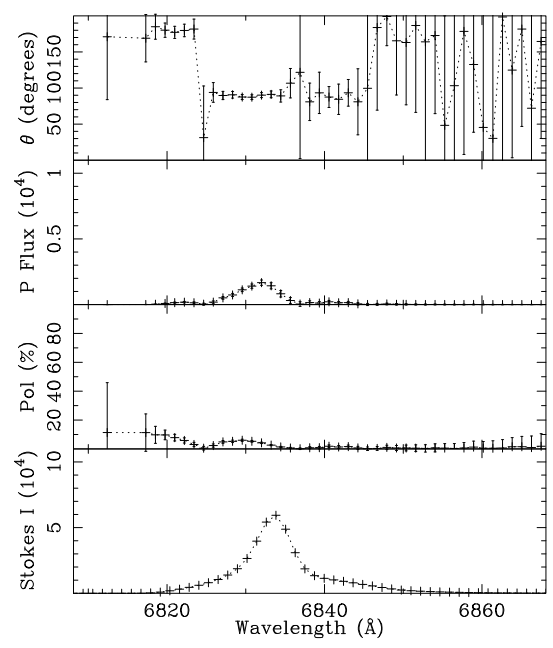


(a)

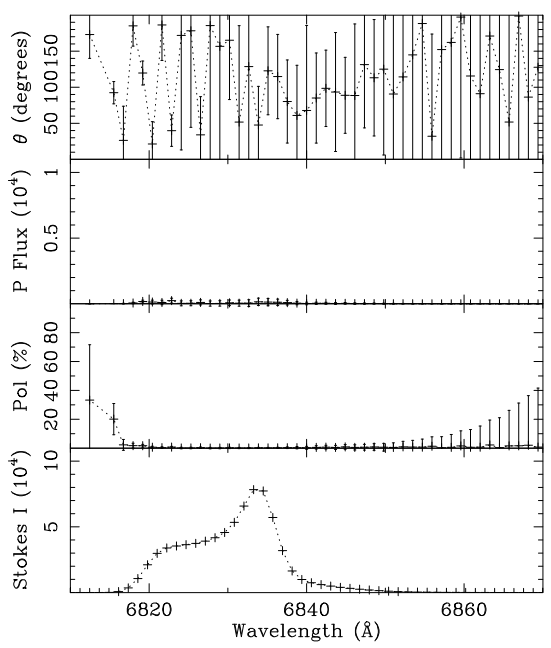

(d)

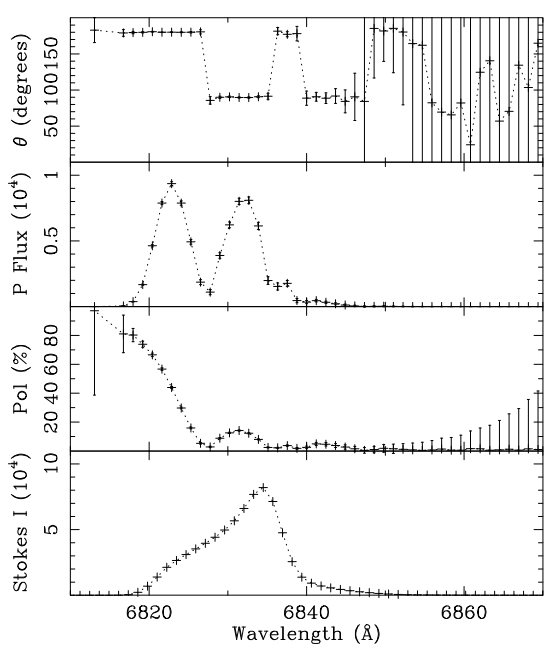

(b)

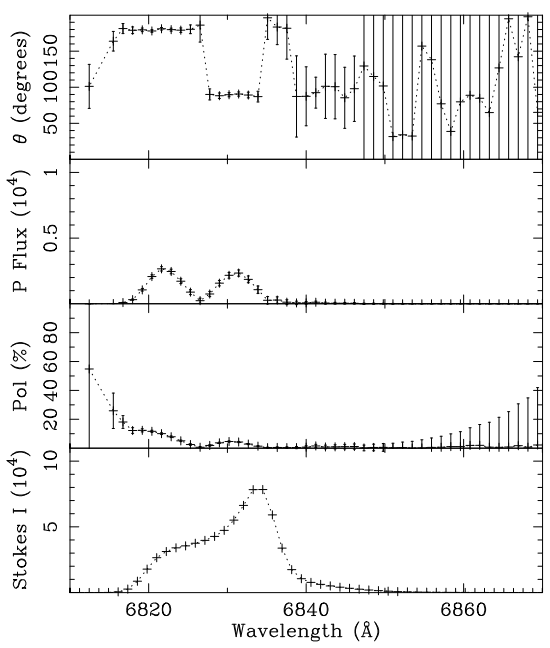

(e)

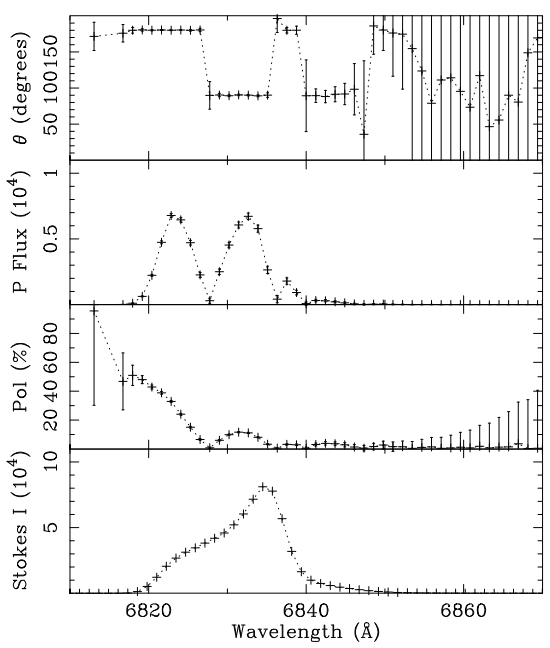

(g)

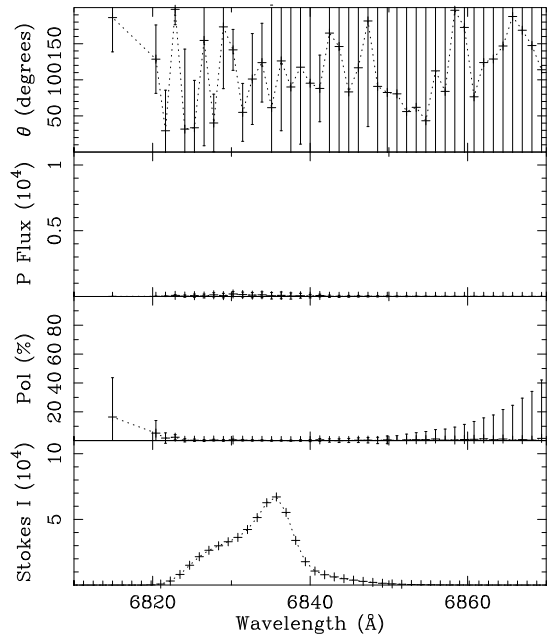

(c)

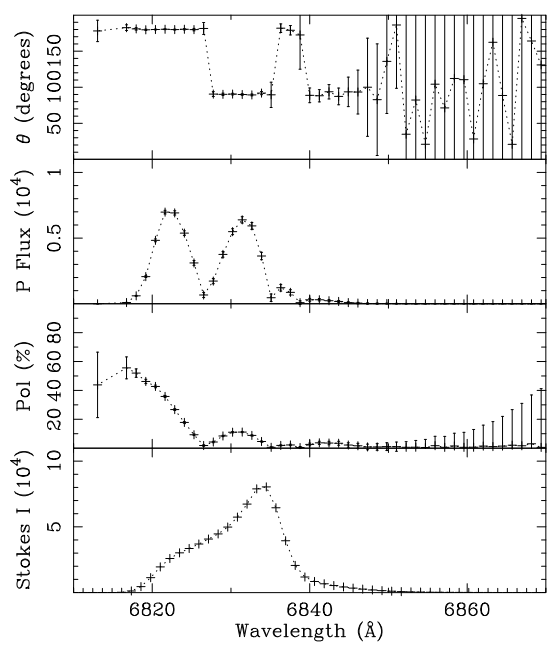

(f)

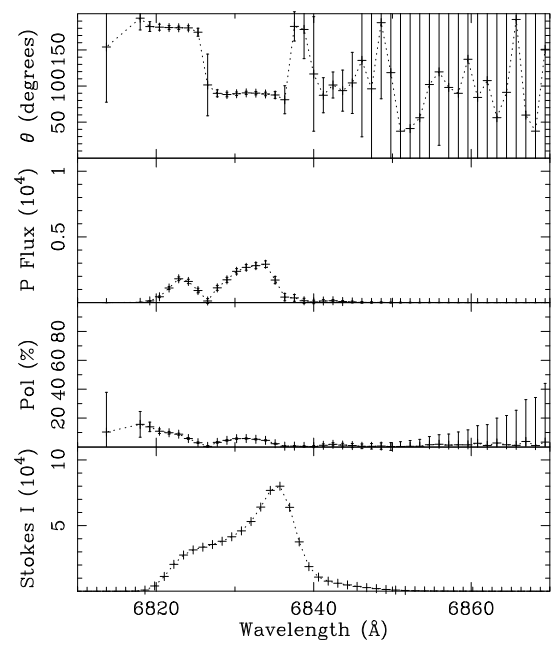




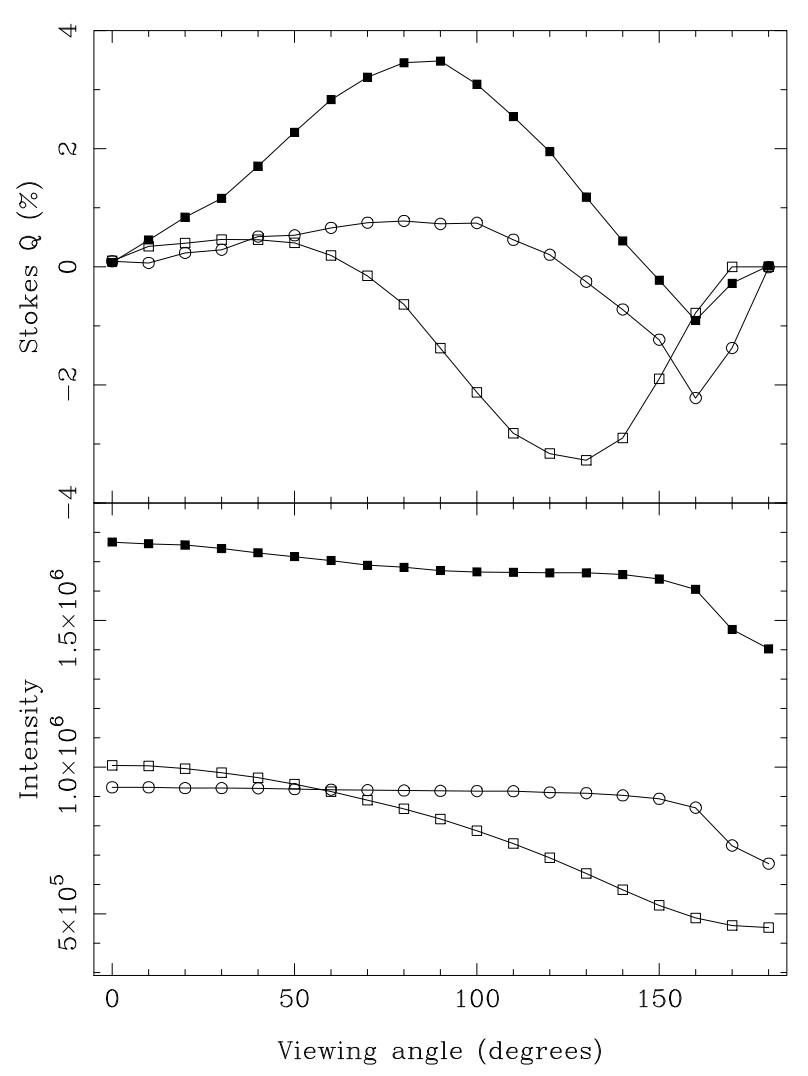

Fig. 21. The integrated line intensity (lower panel) and polarization (upper panel) as a function of phase for models with $\kappa_{\text {red }}=1$ (open squares) and with $\kappa_{\mathrm{UV}}=1$ (open circles). The reference-model results are shown for comparison (filled squares)

Table 4. Quadrature polarization efficiencies and mean velocities for model $\lambda 6825 \AA$ lines

\begin{tabular}{lrr}
\hline Change from reference model & $\eta$ & $\bar{v}$ \\
\hline Reference model & 0.075 & 270 \\
$\lambda 7082 \AA$ & 0.105 & 283 \\
$\log \dot{M}=-5\left(M_{\odot} \mathrm{yr}^{-1}\right)$ & 0.055 & 284 \\
$\log \dot{M}=-7\left(M_{\odot} \mathrm{yr}^{-1}\right)$ & 0.127 & 186 \\
$\log \dot{M}=-8\left(M_{\odot} \mathrm{yr}^{-1}\right)$ & 0.230 & 95 \\
$q_{\mathrm{sep}}=2$ & 0.064 & 311 \\
$q_{\mathrm{sep}}=10$ & 0.090 & 241 \\
$v_{\infty}=10 \mathrm{~km} \mathrm{~s}^{-1}$ & 0.035 & 83 \\
$v_{\infty}=100 \mathrm{~km} \mathrm{~s}^{-1}$ & 0.087 & 446 \\
$v_{(r)}=v_{\infty}\left(1-\left(R_{\mathrm{g}} / r\right)^{2}\right)$ & 0.073 & 260 \\
$\kappa_{\mathrm{Red}}=5$ & 0.050 & 404 \\
$\kappa_{\mathrm{UV}}=5$ & 0.173 & 119 \\
Extended source & 0.045 & 265 \\
Concave ionization & 0.158 & 228 \\
Convex ionization & 0.294 & 188 \\
\hline
\end{tabular}

The quantities $\eta$ and $\bar{v}$ are defined in Eqs. (50) and (51).
1980, Paper I; $N_{\lambda 1032} / N_{\lambda 6825} \sim 12$, Espey et al. 1995). These results are encouraging from the point of view of developing 'tailored' models for individual systems.

The intensity profiles of the observed Raman lines are broadly similar (see Paper I); all the lines are asymmetric, with a stronger blue wing than red. The intensity profiles are often resolved into a multiple subpeak structure, with the most frequently observed profile consisting of a strong central peak with less intense blue- and red-shifted subpeaks. The reference model (Fig. 5) has an asymmetric profile with a stronger blue wing, but does not have resolved subpeaks. As the mass-loss rate is reduced (Fig. 13), the subpeak phenomenon is observed, such as in the reference model with $\log \dot{M}=-7$.

The widths of the observed Raman lines are difficult to measure objectively due to their asymmetric, multipeaked nature and frequently low contrast. Nonetheless, the observed lines are generally much broader than the model profiles obtained by using $v_{\infty}=10 \mathrm{~km} \mathrm{~s}^{-1}$, and are comparable with the models calculated using $v_{\infty}=$ $50-100 \mathrm{~km} \mathrm{~s}^{-1}$. This breadth could result from intrinsically broad O vi lines, but such breadth would 'wash out' the structure in the Raman lines, and would not explain the separation of the observed peaks. In principle, it would be possible to obtain the observed profiles by scattering of suitably-structured O vi emission lines in a static regime. However, this would be entirely ad hoc, would not be consistent with high-dispersion observations of optical emission lines, and would ignore the more natural explanation afforded by scattering in the red-giant wind.

We conclude that the separation of the peaks in the Raman lines is most probably a direct function of the velocity structure of the red-giant wind. In that case the expansion velocities in the scattering regions must typically be closer to $\sim 50 \mathrm{kms}^{-1}$ than the canonical value of $v_{\infty} \approx 10 \mathrm{~km} \mathrm{~s}^{-1}$ frequently adopted for single stars. These two numbers may not be in conflict if the red components of symbiotic systems differ from their isolated counterparts (cf. Kenyon 1988; Whitelock \& Munari 1992), or if most of the acceleration occurs rather close to the star. In the latter case, gravity could very plausibly slow the outflow to the canonical value observed at large distances from single stars, although this would be in conflict with both standard wind theory and the density structure inferred in EG And by Vogel (1991). A direct determination of the far-field velocity of a symbiotic system's red-giant wind would clearly be valuable.

\subsection{Orbital diagnostics}

The most straightforward use of the Raman lines is as binary-orbit diagnostics. All the model polarization spectra show systematic dependences on wavelength which are largely independent of the viewing angle $\alpha$. Thus the rotation of observed PA with binary phase (for orbits with $\cos i \neq 0)$ is virtually model independent; it results solely 
(a)

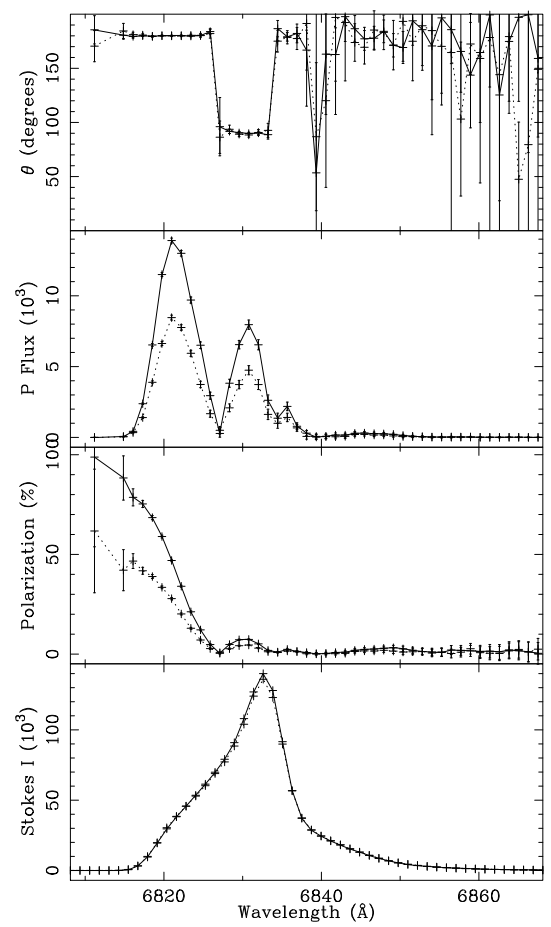

(b)

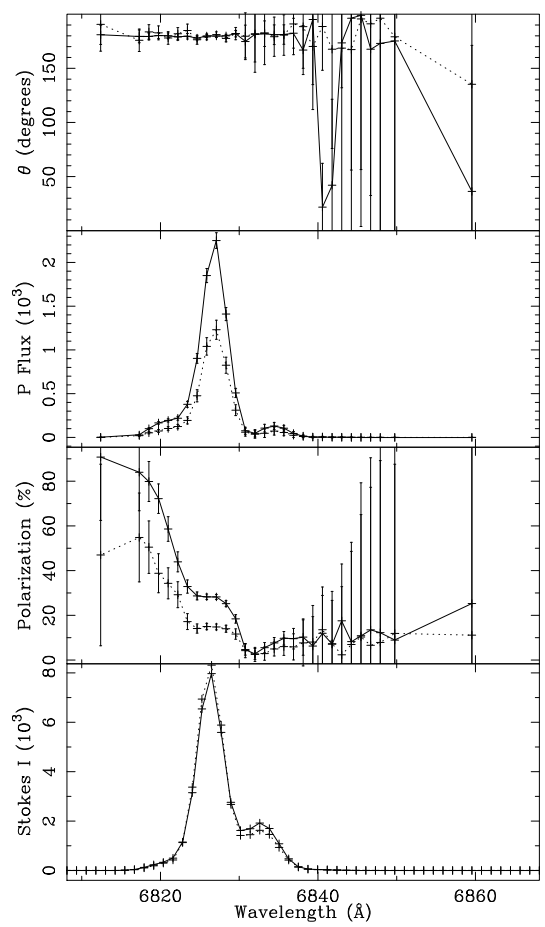

(c)

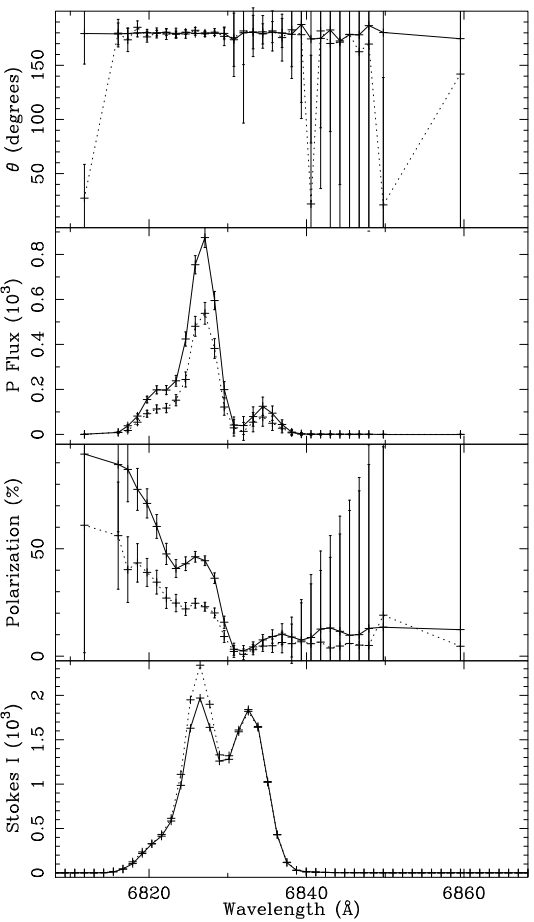

Fig. 22. Polarization profiles calculated using an extended source. a) The reference model (solid line) and a model with $\left.R_{\mathrm{s}}=4 R_{\mathrm{g}}, \mathbf{b}\right)$ the reference model with $\log \dot{M}=-8$ (solid line) and with $\log \dot{M}=-8$ and $R_{\mathrm{s}}=4 R_{\mathrm{g}}$ (dotted line) and c) as for model b) but with $\kappa_{\mathrm{UV}}=1$

from rotation of the binary geometry. Hence a time series of polarization spectra should yield a binary period, simply by observing the period changes in the PA of the polarization.

The magnitude of the Raman-line polarization is a more ambiguous diagnostic, but has some simple properties as a function of binary phase (for systems with $\sin i \neq 0)$. The polarization should reach a maximum twice per binary orbit when the source-scatterer-observer angle is $90^{\circ}$. Similarly, the two polarization minima per period will occur at conjunctions. The models described above show that the Raman-line intensity is much less sensitive to the binary phase than the polarization, particularly in systems of extensive mass-loss. This is a result of the relatively large volume of Raman scattering, which reduces the effect of occultation by the giant star. In systems of lower mass loss the bulk of the scattering occurs in the illuminated hemisphere of the giant and the line intensity shows a strong binary dependence, with the intensity reaching a maximum when the largest fraction of the scattering hemisphere is visible to the observer.

Observations of a small number of objects at two epochs, reported in Paper I, support these predictions of the models. The intensity profiles of the Raman lines are remarkably stable for most objects (with the exception of $\mathrm{RR} \mathrm{Tel,} \mathrm{for} \mathrm{which} \mathrm{the} \mathrm{variation} \mathrm{in} \mathrm{rectified} \mathrm{intensity}$ is probably due to a change in the continuum level rather than a change in the absolute line flux), while the polarization spectra show significant changes. With only two published observations, conclusions cannot be drawn about the binary orbits of these objects, but, in future, timeseries spectropolarimetry will certainly yield valuable data on the symbiotic orbits (cf. Harries \& Howarth 1996a).

\subsection{Failings and future work}

The most troubling features of the model calculations reported here result from scrutiny of the predicted PA structure. The model structure has a quite straightforward interpretation, but the majority of our calculations do not reflect the behaviour reported in Paper I for the majority of systems. In particular, many observations show three (polarized) intensity peaks, with a PA 'flip' in the redmost peak (e.g., Fig. 6); in contrast, most models show a flip in the central peak (e.g., Fig. 5). This may simply be a consequence of the fact that we have concentrated on a region of parameter space most appropriate to relatively close, short-period systems, with negligible 
absorptive opacities; the sensitivity tests do show a rather broad range of behaviour in the models, although none show the 'red flip'.

Qualitatively, we expect that it may be possible to reproduce this PA behaviour in a bipolar-type outflow, with the wind velocity greatest in the direction out of the orbital plane; there is direct evidence that in at least some systems the outflow may be more nearly cylindrically, rather than spherically, symmetric (e.g., Corradi \& Schwartz 1993). The inclusion of a non-spherical wind structure is straightforward in principle, but is once again expensive computationally, since the mass-column calculation requires a numerical approach except for a few special geometries. We have performed a small number of exploratory calculations, but we have not yet succeeded in obtaining a PA flip in only the third (redmost) peak.

A more fundamental difficulty is that any model with 'top-bottom' symmetry can only show emission at position angles of $90^{\circ}$ and $0 / 180^{\circ}$ (or other orthogonal pairs, as the reference system is rotated), because of cancellation in Stokes' U. As demonstrated in Paper I, several systems, such as H2-38, show rotation in PA through the line profiles (that is, loops in the $Q-U$ plane). This rotation can be synthesized within the framework of the models by adding some additional polarized intensity at a different PA. The inference of significant intrinsic continuum polarization in a large fraction of well-observed systems (Paper I) suggests that this is a plausible (if qualitative) explanation. Moreover, rotation is much commoner in the D-type systems discussed in Paper I than in the S-type systems $(6 / 7$, compared to $8 / 17)$. This is circumstantial evidence that dust may implicated, in which case an alignment mechanism would be required.

The basic model contains several assumptions that must be relaxed in later work. The most obvious simplification is the omission of a proper, consistent treatment of the ionized region of the wind. Since Raman scattering arises only in neutral-hydrogen regions, none occur in the $\mathrm{H}$ II wind volume, but the latter will have absorptive and scattering opacities (different to those in the neutral region), including an electron-scattering component, potentially leading to polarization of both Raman and O VI photons; dust scattering and absorption may also be important. The inclusion of the ionized region is conceptually straightforward but requires significantly more computational effort; the intercept between an arbitrary photon path and the boundary region must be calculated repeatedly, and hence an analytical solution must be available if the calculation is to be feasible in reasonable times. In addition, the calculation of the optical depth along a photon path becomes more complex, since absorption and scattering opacities will vary along the photon path. None of these difficulties represent fundamental obstacles.

\subsection{Conclusion}

We have presented the results of Monte-Carlo simulations of Rayleigh+Raman scattering in spherically symmetric, steady-state red-giant winds, with a view to understanding the characteristics of the Raman lines observed in symbiotic systems. These models represent a first reconnaissance of parameter space, and so close matches with specific observations are not to be expected. Nonetheless, encouraging agreement with many observed characteristics has been achieved.

The next phase of this work should be to attempt to relax some of the simplifying assumptions made in this work and to produce 'tailored' models for specific systems. This will be most easily undertaken where repeat observations constrain the orbital parameters and define the phase dependence of the line characteristics. The model described here is sufficiently flexible that it can easily be modified to exploit the additional diagnostic potential offered by the orbital modulation of line strengths resulting from intrasystem line and (quasi-)continuum absorption (cf. Vogel 1991; Shore \& Aufdenberg 1993; Schmid 1995).

Acknowledgements. We thank France Allard and Peter Hauschildt for communicating opacities from their cool-star model atmospheres. The Particle Physics and Astronomy Research Council supported this work through the provision of Starlink computing facilities at UCL, where the numerical work was performed, and of a postgraduate studentship to TJH.

\section{References}

Allen D.A., 1980, MNRAS 190, 87

Bransden B.H., Joachain C.J., 1983, Physics of Atoms and Molecules (Longman)

Chandrasekhar S., 1960, Radiative Transfer (Dover)

Corradi R.L., Schwartz H.E., 1993, A\&A 268, 714

Espey B.R., Schulte-Ladbeck R.E., Kriss G.A., et al., 1995, ApJ 454, L61

Harries T.J., Howarth I.D., 1996a, A\&A 310, 235

Harries T.J., Howarth I.D., 1996b, A\&AS 119, 61 (Paper I)

Kenyon S.J., 1988, AJ 96, 337

Kenyon S.J., Fernandez-Castro T., 1987, AJ 93, 938

Kindl C., Marxer N., Nussbaumer H., 1982, A\&A 116, 265

Loup C., Forveille T., Omont A., Paul J.F., 1993, A\&AS 99, 291

Michalitsianos A.G., Kafatos M., Fahey R.P., Viotti R., Cassatella A., Altamore A., 1988, ApJ 331, 477

Mürset U., Nussbaumer H., Schmid H.M., Vogel M., 1991, A\&A 248, 458

Nussbaumer H., Schmutz W., Vogel M., 1995, A\&A 293, L13

Nussbaumer H., Vogel M., 1987, A\&A 182, 51

Penston M.V., Benvenuti P., Cassatella A., et al., 1983, MNRAS 202, 833

Press W.H., Flannery B.P., Teukolsky S.A., Vetterling W.T., 1989, Numerical Recipes (CUP) 
Schmid H.M., 1989, A\&A 221, L31

Schmid H.M., 1992, A\&A 254, 224

Schmid H.M., 1995, MNRAS 275, 227

Schmid H.M., Schild H., 1990, A\&A 236, L13

Schmid H.M., Schild H., 1994, A\&A 281, 145

Schmutz W., Schild H., Mürset U., Schmid H.M., 1994 A\&A 288,819
Seaquist E.R., Taylor A.R., Button S., 1984, ApJ 184, 202 (erratum 317, 555)

Seaquist E.R., Taylor A.R., 1990, ApJ 349, 313

Shore S.N., Aufdenberg J.P., 1993, ApJ 416, 355

Viotti R., Ricciardi O., Ponz D., et al., 1983, A\&A 119, 285

Vogel M., 1991, A\&A 249, 173

Whitelock P.A., Munari U., 1992, A\&A 255, 171 